\title{
Inspecting gradual and abrupt changes in emotion dynamics with the time-varying change point autoregressive model
}

\author{
Casper J. Albers* and Laura F. Bringmann* \\ * Joint first author \\ University of Groningen
}

\begin{abstract}
Recent studies have shown that emotion dynamics such as inertia (i.e., autocorrelation) can change over time. Importantly, current methods can only detect either gradual or abrupt changes in inertia. This means that researchers have to choose a priori whether they expect the change in inertia to be gradual or abrupt. This will leave researchers in the dark regarding when and how the change in inertia occurred. Therefore in this article we use a new model: the time-varying change point autoregressive (TVCP-AR) model. The TVCP-AR model can detect both gradual and abrupt changes in emotion dynamics. More specifically, we show that the inertia of positive affect and negative affect measured in one individual differ qualitatively in how they change over time. Whereas the inertia of positive affect increased only gradually over time, negative affect changed both in a gradual and abrupt fashion over time. This illustrates the necessity of being able to model both gradual and abrupt changes in order to detect meaningful quantitative and qualitative differences in temporal emotion dynamics.
\end{abstract}

\section{The Supplementary Materials are appended to this manuscript file.}

Keywords: Dynamic modeling, Change point detection, Generalized additive modeling, Inertia, Emotion dynamics

\section{Introduction}

Whereas your height will not change in a couple of days or even years, your emotions will. In fact, both due to internal (e.g., biological rhythms) and external factors (e.g., social interactions), emotional fluctuations occur within a day or even an hour (Kuppens \& Verduyn, 2015). These fluctuations or emotion dynamics call for methods that can assess emotional experience across time at small enough intervals, such as ambulatory assessment, experience sampling method, and ecological momentary assessment (Houben, Van Den Noortgate, \& Kuppens, 2015; Trull \& Ebner-Priemer, 2013; Trull, Lane, Koval, \& Ebner-Priemer, 2015). With these methods, one can measure emotional fluctuations at different time in-

Heymans Institute for Psychological Research, University of Groningen, Grote Kruisstraat 2/1, 9712 TS Groningen, The Netherlands. Contact: 1.f.bringmann@rug.nl.

We thank the editor and two anonymous reviewers for their input and Markus Eronen for helpful comments on earlier versions of the manuscript. The Center for Information Technology of the University of Groningen provided access to the Peregrine high performance computing cluster.

Laura Bringmann's research was supported by the Netherlands Organization for Scientific Research (NWO; Veni grant 191G.037). tervals, for example, every day, hour, or even every minute, leading to what is known as intensive longitudinal data (Bolger \& Laurenceau, 2013; Ebner-Priemer \& Trull, 2009; Walls \& Schafer, 2006)

Importantly, this kind of intensive longitudinal data has shown that not only how people feel on average (i.e., mean level of one's emotion) but also the temporal dynamics of emotions is key information for a person's well-being, (Chow, Ram, Boker, Fujita, \& Clore, 2005; Kuppens, Champagne, \& Tuerlinckx, 2012; Verduyn, Van Mechelen, Tuerlinckx, Meers, \& Van Coillie, 2009). Thus, while it is certainly essential information to know if a person has on average a high negative affect, the pattern of how negative affect changes over time is also thought to give crucial information about a persons well-being (Trull et al., 2015).

One dynamic feature of emotions that has been of special interest in recent emotion research is temporal dependency or inertia (Kuppens et al., 2012; Suls, Green, \& Hillis, 1998; Suls \& Martin, 2005). Inertia can be defined as how resistant an emotion is to change. For instance, if an emotion has a high predictability over time it is likely that one stays in a certain emotion and thus that the emotion has a high spillover from one moment to the next. High inertia has therefore been defined as a decrease in the ability of a person to have emotional changes and thus as less well working (or maladaptive) emotion-regulation skills (Kuppens, Allen, \& 
Sheeber, 2010; Kuppens \& Verduyn, 2017). In line with this reasoning, several studies showed a positive association between high inertia and depressive symptoms or neuroticism (e.g., Brose, Schmiedek, Koval, \& Kuppens, 2015; Koval, Kuppens, Allen, \& Sheeber, 2012; Koval, Pe, Meers, \& Kuppens, 2013; Suls et al., 1998; Wenze, Gunthert, Forand, \& Laurenceau, 2009, see, however, Dejonckheere et al. 2019).

Formally, inertia can be calculated through an autocorrelation of an emotion, or by fitting, as is commonly done, an autoregressive (multilevel) model (Jahng, Wood, \& Trull, 2008; Krone, Albers, Kuppens, \& Timmerman, 2017; Rovine \& Walls, 2006; Schuurman, Ferrer, de Boer-Sonnenschein, \& Hamaker, 2016). The drawback of these models, however, is that they assume stationarity. This implies, for instance, that the average value around which an emotion is fluctuating and its temporal dependency (i.e., autocorrelation or inertia) is time-invariant (Chatfield, 2003; Hamaker, Ceulemans, Grasman, \& Tuerlinckx, 2015). This stationarity assumption is problematic as studies have shown that inertia does in fact change over time. For example, Koval and Kuppens (2012) have shown that emotional inertia changes due to a social stressor. Furthermore, Bringmann et al. (2017) showed that inertia can change in an individual brought into social isolation.

Even more significantly, current theories stemming from dynamical systems theory (Scheffer et al., 2009) suggest that changes in symptoms or emotion dynamics are crucial for predicting and explaining how individuals develop psychiatric disorders (van de Leemput et al., 2014). More specifically, 'critical slowing down' that is signaled by an increase in the autocorrelation of the symptoms or emotions of an individual could function as an 'early warning signal' before an individual transitions from a healthy state, to, for instance, a state of depression (Nelson, McGorry, Wichers, Wigman, \& Hartmann, 2017; Wichers, Groot, Psychosystems, ESM Group, \& EWS Group, 2016). In this case, the person has more difficulty in recovering from perturbations and thus regulating her/his emotions (Cabrieto, Adolf, et al., 2018; Cramer et al., 2016). Importantly, the type of change plays a key role in these hypotheses about how individuals develop psychiatric disorders. Specifically, a gradual increase in the autocorrelation could be an important warning signal that precedes an abrupt change in which an individual transitions into a depression. Wichers et al. (2016) found initial support for such warning signals. An increase in the autocorrelation of several mood states was found before an individual transitioned into a depression after medication reduction.

This shows the need for statistical methods that can detect and model changes in the temporal emotion dynamics and thus changes in the autocorrelation over time. Although statistical developments have led to methods that can model changes in the autocorrelation, each of these methods can model only one type of change; either gradual (e.g.,
Bringmann, Ferrer, Hamaker, Borsboom, \& Tuerlinckx, 2018; Chow et al., 2005; Chow, Zu, Shifren, \& Zhang, 2011; Haslbeck \& Waldorp, 2018; Molenaar, De Gooijer, \& Schmitz, 1992) or abrupt change (e.g., Cabrieto, Tuerlinckx, Kuppens, Grassmann, \& Ceulemans, 2017; Cabrieto, Tuerlinckx, Kuppens, Hunyadi, \& Ceulemans, 2018; Hamaker \& Grasman, 2012). However, the models mentioned here assume gradual change and will not be able to detect or represent abrupt changes, and vice versa. This will leave researchers in the dark regarding when and how the change in autocorrelation occurred. Therefore, in order for a model to be applicable to detect changes in emotion dynamics, it is crucial that it is able to model both change processes, gradual and abrupt.

In this paper we will introduce a new model that can detect both gradual and abrupt change in temporal emotion dynamics: the time-varying change point autoregressive (TVCP-AR) model. As a starting point this uses the timevarying autoregressive (TV-AR) model and combines it with a change point $(\mathrm{CP})$ modelling approach (Bringmann et al., 2017; Hamilton, 1989).

The outline of this paper is as follows. In the next section, we will discuss these TV-AR and CP models. This is followed by an explanation of the TVCP-AR model. After this, we will showcase the TVCP-AR model with an experience sampling study of a single patient who underwent medication reduction. We end with a discussion of the possibilities and limitations of the TVCP-AR model for future research in emotion dynamics. In the Supplementary Material, we provide technical details and show through an extensive simulation that our model performs well in circumstances common for psychological research.

\section{The two models that are the ingredients of the TVCP-AR model}

\section{The TV-AR model}

The TV-AR model is an extension of the AR(1) model, where the parameters can vary over time. Non-stationarity can thus be explicitly dealt with, meaning that a person's inertia is allowed to change over time. This TV-AR model is defined by

$$
y_{t}=\beta_{0, t}+\beta_{1, t} y_{t-1}+\varepsilon_{t}, \quad t>1 .
$$

In this model, both the intercept, $\beta_{0, t}$, and slope, $\beta_{1, t}$ are allowed to change gradually over time. There are various ways to impose that changes of the parameters occur gradually. This model is based on the generalized additive model (GAM) framework (Bringmann et al., 2017; Dahlhaus, 1997; Giraitis, Kapetanios, \& Yates, 2014; Wood, 2006). Here, the estimation of $\beta_{0, t}$ and $\beta_{1, t}$ is done via nonparametric smooth functions based on regression splines (Hastie \& Tibshirani, 1990). 
As in all models with smoothing, there is a trade-off between model fit and smoothness of the resulting estimates. In the TV-AR model, the optimal level of smoothness is derived via generalized cross-validation (Golub, Heath, \& Wahba, 1979). In practice, estimation is straightforward using the $m c g v$ package in $R$ (Wood, 2006).

\section{The change point model}

Especially within the econometric literature, there is an abundance of models for modelling time series where the values of the parameters depend on the state, or regime, one is in. In the simplest case, one works with two regimes. Following Hamilton $(1989,1994)$, this model has the form

$$
y_{t}= \begin{cases}\beta_{0}+\beta_{1} y_{t-1}+\varepsilon_{t} & t \leq C P \\ \beta_{0}+\delta+\beta_{1} y_{t-1}+\varepsilon_{t} & t>C P .\end{cases}
$$

Thus, up to a certain change point $C P$, the intercept of this model is $\beta_{0}$, and after the change point it is $\beta_{0}+\delta$. Such a model with one or more abrupt changes is known as a structural change point model. In the formulation of Hamilton, the regimes before and after the change point are only different with respect to the value of the intercept, but it is straightforward to extend this to also allow for differences in the autoregressive effects. The model Hamilton specified is the basis for a broad class of models, including change point and regime switching models that are already applied in emotion research (e.g., Cabrieto et al., 2017; Cabrieto, Tuerlinckx, et al., 2018; de Haan-Rietdijk, Gottman, Bergeman, \& Hamaker, 2014; Hamaker \& Grasman, 2012).

\section{The TVCP-AR model}

The TV-AR model allows for smooth variation of the dynamics in an $\mathrm{AR}(1)$ process, but not for sudden changes. The structural change point model, on the other hand, does not allow for smooth changes but does allow for sudden changes. By combining these two models, we introduce the time-varying change point AR(1) model (TVCP-AR(1)), that allows for both smooth and sudden changes in the dynamics.

The sudden change can be either at an expected moment, such as the start of treatment, or a specific moment which can be pinpointed in hindsight, such as the occurrence of a life event. In those cases, the confirmatory TVCP-AR model can be used to check whether there was indeed a (significant) change at that time point and, if so, how big the change was (see Supplementary Material). It is, however, also possible that the sudden change takes place at some unexpected moment. In order to find such a sudden change, the exploratory TVCP-AR model can be used.

Without knowledge of where the change takes place, one should check all possible options. A change point model partitions the data into two periods: the one up to the switch and the one after the switch. For an exploratory search towards the location of the change point, we use the following algorithm.

First, fit a TV-AR model without change points and denote the corresponding Akaike Information Criterion (AIC) value by $\mathrm{AIC}^{0}$. As a next step, for each $2<i<T-1$, perform the following actions:

1. Fit a model with a change point at location $i$ to the data;

2. Denote the AIC-value of this model by $\mathrm{AIC}_{i}^{1}$.

Let $\mathrm{AIC}^{1}=\operatorname{argmin}_{i} \mathrm{AIC}_{i}^{1}$ and denote the value $i$ for which this minimum is attained by $j$. Compute $\mathrm{AIC}^{1}-\mathrm{AIC}^{0}$. If the improvement in AIC is too small there is no indication for a change point. If, however, the $\mathrm{AIC}^{1}$ score lies substantially below $\mathrm{AIC}^{0}$, then location $j$ will be denoted as the first switch point.

The simulations in the Supplementary Material suggest that to avoid too many false positives, a reasonable threshold for the AIC difference is somewhere between -10 and -15 . However, setting the threshold is in the end a trade-off between power and false positive rate, and depends on the number of time points and the expected effect size. Note that rather than the AIC, other information criteria, such as the Bayesian Information Criterion (BIC), can be also applied. In the Supplementary Material, we study model selection based on both AIC and BIC and provide advice on which one to choose and which threshold to choose with it.

Note that it is undesirable to allocate change points at extreme ends of the time series. When, for instance, a change point would be allocated at $t=2$, this implies that the first regime only has a single observation. Putting an timevarying autoregressive model on one or two observations is clearly undesirable, thus rather than applying the approach above for $2<i<T-1$, it makes more sense to apply it only to $k<i<T-k+1$, for some small value of $k$ (e.g. $k=3$ ).

Once a change point has been found, the time series is partitioned into two parts. For each part, a TV-AR model is fitted, allowing also for a gradual change in the AR(1) coefficients. Furthermore, the above strategy above can be applied again to find a new change point. This approach can be repeated until no further change points are detected. Figure 1 sketches the approach suggested here. A detailed explanation of this model is provided in the Supplementary Material.

\section{Empirical example}

In order to illustrate the TVCP-AR model, we analysed data of one patient from an experience sampling study of 239 days (Wichers et al., 2016; see also Kossakowski, De Groot, Haslbeck, Borsboom, \& Wichers, 2017). During this study, the patient (with major depression) underwent medication reduction, and was beeped 10 times per day to report on momentary experiences, resulting in 1,474 time points. First there was a baseline period of 28 days. After this, the trial period started (days 29-127), with medication reduction starting at day 42 . This was followed by a post-trial assess- 


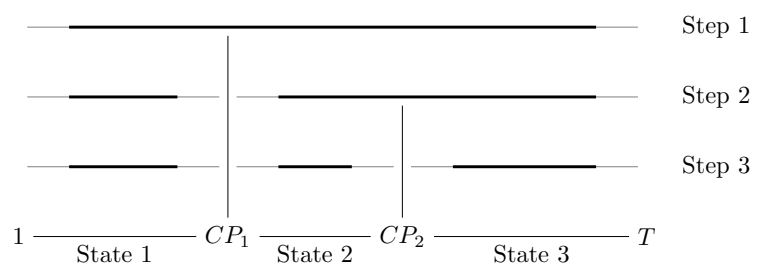

Figure 1. The exploratory procedure for finding multiple change points. Step 1 starts with the full time series $1, \ldots, T$ and excludes a few observations at each end (depicted by a thin grey line) and searches for a change point in the remaining observations. One is found at location $C P_{1}$. In Step 2 the time series is partitioned in two: $1, \ldots, C P_{1}$ and $C P_{1}+1, \ldots, T$. Both partitions have their buffer zones and in both partitions new change points are sought. No change point is found in $1, \ldots, C P_{1}$, but one is found in $C P_{1}+1, \ldots, T$ at location $C P_{2}$. This subdivides this second partition in two partitions and in Step 3 these are searched for a change point. In neither of them one is found, thus the model splits the time series into three states.

ment period (days 129-155), and finally an additional assessment period (days 156-239; see Figure 2). In this study, it was found based on the SCL-90-R depression subscale that this patient had a sudden increase in depression symptoms around day 127 . Here we only analyze pre-processed data for variables positive affect and negative affect (see Figure 2, Panel (a) and (b)). Besides Wichers et al. (2016), the same data has been previously analyzed by Cabrieto, Tuerlinckx, et al. (2018). Both studies indeed detected a 'critical slowing down' preceding the relapse, signaled by an increase in the autocorrelation of positive and negative effect. However, these studies used analyses that could detect either gradual or abrupt changes in the autocorrrelation, but not both kinds of change simultaneously.

In order to be able to compare our study with the studies of Wichers et al. (2016) and Cabrieto, Tuerlinckx, et al. (2018), we used the detrended version of the data, and excluded time points for which lag 1 counterparts were not available, as well as those that were preceded by a night. The data and $\mathrm{R}$-code to replicate the TVCP-AR analyses can be found in the Supplementary Material. To get an indication of the robustness of our results we used both the AIC and BIC for model selection. Following the recommendation based on our simulation study in the Supplementary Material, we set a threshold of -15 on AIC or BIC difference for labeling the finding as evidence for a change point in the data. We used such a strict threshold in order reduce the risk of discovering false positives. All analyses of the empirical example can be found online as $R$-files.

\section{Results}

As the TVCP-AR is a univariate model, we analyzed positive affect and negative affect separately. Starting with positive affect, the TVCP-AR model clearly shows that there is hardly any difference in AIC from the model without to the model with change point (see Figure 2, panel (c). The largest difference in AIC is 1.68, which is clearly less than 15. Figure 2 panel (d) shows the final model, which is in essence a TV-AR(1) model with a slow gradual change in autocorrelation, with no evidence of an abrupt change. Using the BIC led to the same results.

In contrast, in the negative affect data both gradual and abrupt changes can be found. Both the AIC and BIC indicated a change point at day 47 (with an AIC difference of -20.33 and a BIC difference of -20.26; see also the Supplementary Material). A second change point was also detected, but its exact timing was less robust. Using the AIC, the change point was found around 120 days (with an AIC difference of 22.81; see Figure 2 panel (e)), but also a further change point was detected around day 127, thus the same day around which the patient relapsed into depression. This change point was extremely close to the change point at day 120. Therefore, there were not enough time points between the change points at day 120 and day 127 to perform the next step of the TVCP-AR(1) model, in which the gradual change in the autocorrelation is modelled. ${ }^{1}$ The BIC, on the other hand, indicated a BIC difference of -17.90 for a change point at day 106.

As a general pattern, the change points occurred after the medication dose reduction. There was a gradual increase of the autocorrelation before day 47, after which the autocorrelation abruptly dropped. A second increase in autocorrelation was detected before the patient relapsed into depression. The exact timing of this increase in the autoregressive parameter, however, could not be robustly determined. Based on Figure 2 panel (f), a gradual increase seemed to have started around day 80 , whereas an abrupt change was detected around day 106, 120 or 127 . This overall period overlaps with the timing where Cabrieto, Tuerlinckx, et al. (2018) detected a change point (day 86).

\section{Discussion}

There is increasing evidence that emotion dynamics such as inertia change over time. Especially the literature on complex system dynamics in psychopathology calls for methods that can detect early warning signals, such as when and how changes in inertia or autocorrelation occur (Wichers, Schreuder, Goekoop, \& Groen, 2019). Whereas current methods can only detect either gradual or abrupt changes in autocorrelation, we showed in this article how the TVCP-AR

\footnotetext{
${ }^{1}$ Both patterns show first an increase and then a decrease in autocorrelation.
} 
(a)

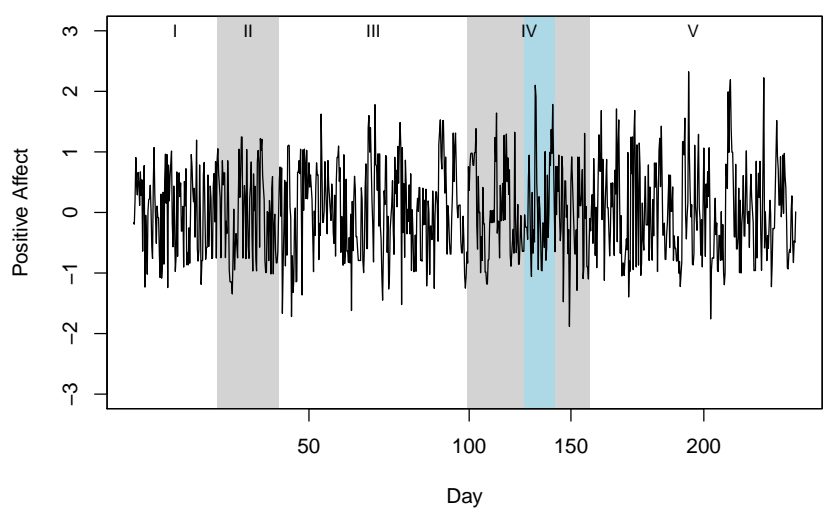

(r)

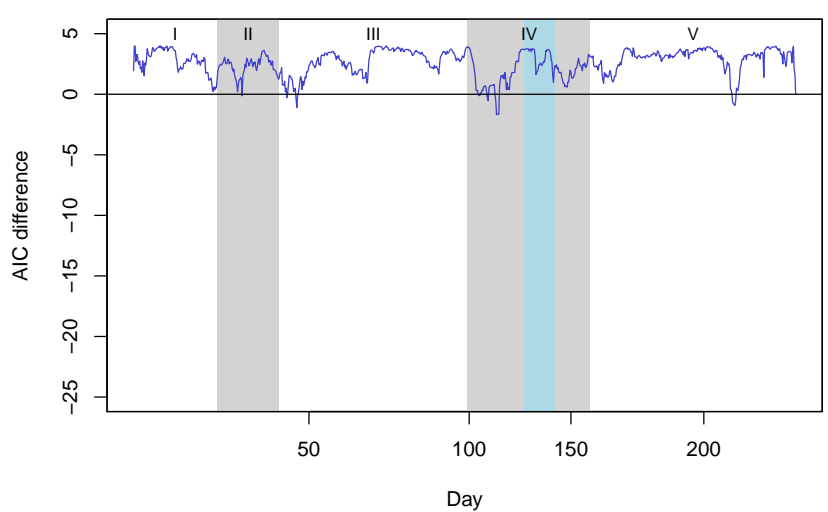

(a)

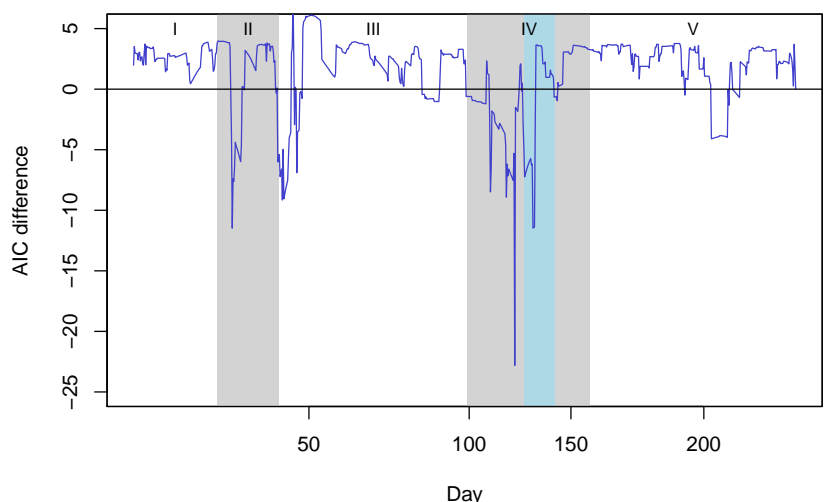

(b)

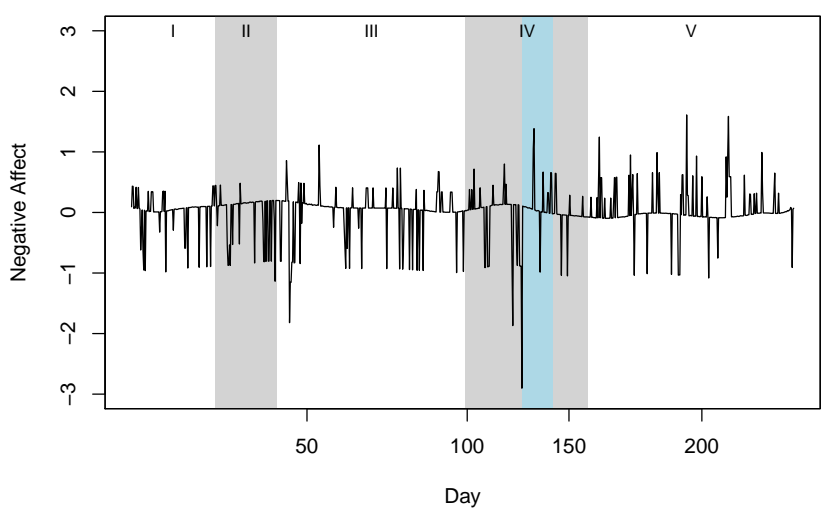

(d)

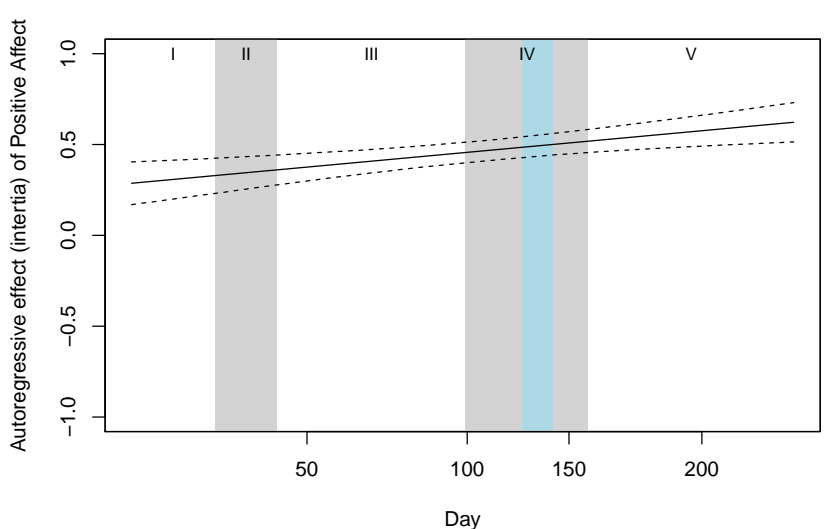

(f)

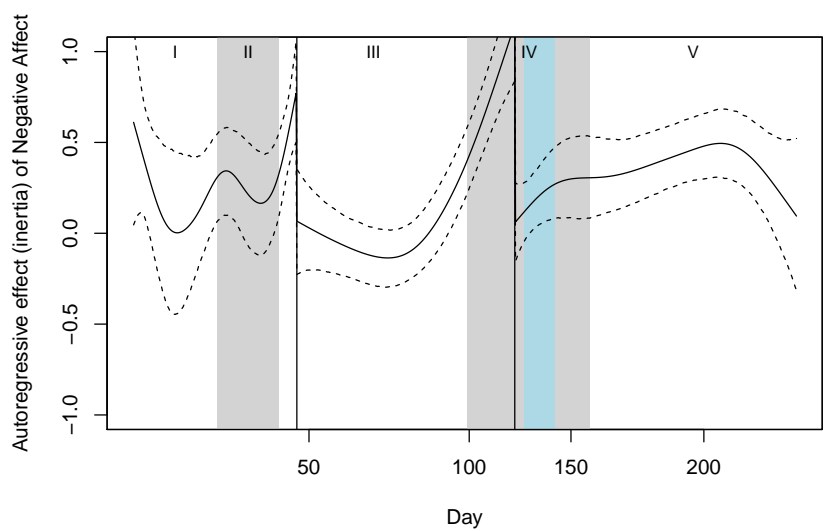

Figure 2. Panel (a) raw detrended Positive Affect scores; (b) raw detrended Negative Affect scores; (c) AIC difference, positive affect; (d) final model for inertia of positive affect; (e) AIC difference of negative affect for the first change point; (f) final model of inertia for negative effect. I = Baseline, II = Before Dose Reduction, III = Dose Reduction; IV = Post Assessment; V = Follow-up. The blue vertical line represents the week in which the clear increase in the severity of the symptoms occurred. Note that the days on the horizontal axis are not equally spaced. This reflects the missingness in the data: For example, between day 100 and day 150 there were more missing values than between day 50 and day 100 . 
model can detect both gradual and abrupt changes in emotion dynamics. More specifically, we showed that in the empirical example the autocorrelations of positive and negative affect differ qualitatively in how they change over time. Whereas the autocorrelation of positive affect increases only gradually over time, negative affect changes both in a gradual and abrupt fashion. This application, thus, illustrates the necessity of modeling both gradual and abrupt changes in order to detect meaningful quantitative and qualitative differences in temporal emotion dynamics.

Importantly, the TVCP-AR is not suitable for all kinds of qualitative changes. Change point analysis as such is especially suited when abrupt changes happen several times, but unsuited when the emotion dynamics are shifting frequently between different regimes for short times. In this case, regime switching models should be used (see, for example, Hamaker, 2009). Additionally, TVCP-AR model is a discrete time model, which limits its ability to deal with unequally spaced time points (Ryan, Kuiper, \& Hamaker, 2019; Voelkle \& Oud, 2013). Developing continuous forms of the TVCP-AR model would be a fruitful endeavor (e.g., along the lines of Chen, Chow, \& Hunter, 2019).

Another limitation of the TVCP-AR model is that an extensive number of time points is needed in order to find gradual and abrupt changes. In general, when the effect of the abrupt change is pronounced, around 100 time points are needed (see Supplementary Material). However, this number increases when there is both a gradual and abrupt change or if the change in dynamics is not pronounced. A related issue is that a TVCP-AR can indicate, as in the empirical example, that two change points are fairly close to one another. In this case, the TVCP-AR could not give information on the period between these change points.

An advantage of the TVCP-AR model is that, in contrast to for example the method suggested by Cabrieto, Tuerlinckx, et al. (2018), one can distinguish between changes in the mean or the autocorrelation of the process. In the empirical example, the data was already detrended, but with the TCVP-AR model such detrending as a preprocessing step is not necessary. Instead, the TVCP-AR model can distinguish whether changes happen in the mean or the autocorrelation of the process under study (or in both). This is an important advantage, as recently there has been debate regarding the predictive value of dynamic measures such as the autoregressive coefficient over and above the mean in the context of emotion dynamic research (Dejonckheere et al., 2019).

On top of allowing for dynamic changes in mean level and inertia, our model allows for abrupt changes in the variability: for each segment (see Figure 1) a separate variance is modelled for the error terms $\varepsilon_{t}$. Changes in intraindividual variability can be indicators of psychopathological importance (Du \& Wang, 2018). Within the current GAMframework, it is unfortunately not possible to allow for grad- ual changes in the variation on top of the modelled abrupt changes.

The idea of early warning signals has also been incorporated into the psychological network approach (Cramer et al., 2016). In this approach, the main focus of interest is not on the inertia or autocorrelation of an emotion, but rather on the interaction between emotions or symptoms (Borsboom \& Cramer, 2013). For instance, Wichers et al. (2016) showed using the same empirical dataset that not just the autocorrelations of the mental states, but also interactions between the mental states (i.e., the dynamic network) increased in strength near the transition. Current network models, however, are either based on a vector autoregressive model (Pe et al., 2015; Wigman et al., 2015), in which no change over time can be modeled, or on time-varying vector autoregressive models that can only model gradual change over time (Bringmann et al., 2018; Haslbeck, Bringmann, \& Waldorp, 2018; Haslbeck \& Waldorp, 2018). A multivariate extension of the TVCP-AR with which also the dynamics between emotions can be modeled in a gradual and abrupt is currently under development. In sum, the TVCP-AR is a powerful method for modeling and detecting different types of changes in emotion dynamics.

\section{References}

Bolger, N., \& Laurenceau, J.-P. (2013). Intensive longitudinal methods: An introduction to diary and experience sampling research. New York, NY: Guilford Press.

Borsboom, D., \& Cramer, A. O. (2013). Network analysis: an integrative approach to the structure of psychopathology. Annual Review of Clinical Psychology, 9, 91-121.

Bringmann, L. F., Ferrer, E., Hamaker, E. L., Borsboom, D., \& Tuerlinckx, F. (2018). Modeling nonstationary emotion dynamics in dyads using a time-varying vector-autoregressive model. Multivariate behavioral research, 53(3), 293-314.

Bringmann, L. F., Hamaker, E. L., Vigo, D. E., Aubert, A., Borsboom, D., \& Tuerlinckx, F. (2017). Changing dynamics: Time-varying autoregressive models using generalized additive modeling. Psychological Methods, 22(3), 409-425. doi: $10.1037 /$ met0000085

Brose, A., Schmiedek, F., Koval, P., \& Kuppens, P. (2015). Emotional inertia contributes to depressive symptoms beyond perseverative thinking. Cognition and Emotion, 29(3), 527538.

Cabrieto, J., Adolf, J., Tuerlinckx, F., Kuppens, P., Ceulemans, E., \& Cabrieto, J. (2018). An objective, comprehensive and flexible statistical framework for detecting early warning signs of mental health problems. Psychotherapy and psychosomatics, $1-3$.

Cabrieto, J., Tuerlinckx, F., Kuppens, P., Grassmann, M., \& Ceulemans, E. (2017). Detecting correlation changes in multivariate time series: A comparison of four non-parametric change point detection methods. Behavior research methods, 49(3), 988-1005.

Cabrieto, J., Tuerlinckx, F., Kuppens, P., Hunyadi, B., \& Ceulemans, E. (2018). Testing for the presence of correlation 
changes in a multivariate time series: A permutation based approach. Scientific reports, 8(1), 769.

Chatfield, C. (2003). The analysis of time series: An introduction (Fifth ed.). Boca Raton, FL: Chapman and Hall/CRC

Chen, M., Chow, S.-M., \& Hunter, M. D. (2019). Stochastic differential equation models with time-varying parameters. In Continuous time modeling in the behavioral and related sciences (pp. 205-238). Springer.

Chow, S.-M., Ram, N., Boker, S. M., Fujita, F., \& Clore, G. (2005). Emotion as a thermostat: representing emotion regulation using a damped oscillator model. Emotion, 5(2), 208.

Chow, S.-M., Zu, J., Shifren, K., \& Zhang, G. (2011). Dynamic factor analysis models with time-varying parameters. Multivariate Behavioral Research, 46(2), 303-339.

Cramer, A., van Borkulo, C., Giltay, E., van der Maas, H., Kendler, K., Scheffer, M., \& Borsboom, D. (2016). Major depression as a complex dynamical system. PLoS ONE, 11(12), e0167490. doi: 10.1371/journal.pone.0167490

Dahlhaus, R. (1997). Fitting time series models to nonstationary processes. The Annals of Statistics, 25(1), 1-37.

de Haan-Rietdijk, S., Gottman, J. M., Bergeman, C. S., \& Hamaker, E. L. (2014). Get over it! A multilevel threshold autoregressive model for state-dependent affect regulation. Psychometrika, 1-25.

Dejonckheere, E., Mestdagh, M., Houben, M., Rutten, I., Sels, L., Kuppens, P., \& Tuerlinckx, F. (2019). Complex affect dynamics add limited information to the prediction of psychological well-being. Nature human behaviour, 3(5), 478.

Du, H., \& Wang, L. (2018). Reliabilities of intraindividual variability indicators with autocorrelated longitudinal data: Implications for longitudinal study designs. Multivariate Behavioral Research, 53(4), 502-520. doi: 10.1080/00273171.2018.1457939

Ebner-Priemer, U. W., \& Trull, T. J. (2009). Ambulatory assessment: An innovative and promising approach for clinical psychology. European Psychologist, 14(2), 109-119.

Giraitis, L., Kapetanios, G., \& Yates, T. (2014). Inference on stochastic time-varying coefficient models. Journal of Econometrics, 179(1), 46-65.

Golub, G. H., Heath, M., \& Wahba, G. (1979). Generalized crossvalidation as a method for choosing a good ridge parameter. Technometrics, 21(2), 215-223.

Hamaker, E. L. (2009). Using information criteria to determine the number of regimes in threshold autoregressive models. Journal of Mathematical Psychology, 53(6), 518-529.

Hamaker, E. L., Ceulemans, E., Grasman, R., \& Tuerlinckx, F. (2015). Modeling affect dynamics: State of the art and future challenges. Emotion Review, 7(4), 316-322.

Hamaker, E. L., \& Grasman, R. (2012). Regime switching statespace models applied to psychological processes: Handling missing data and making inferences. Psychometrika, 77(2), 400-422.

Hamilton, J. D. (1989). A new approach to the economic analysis of nonstationary time series and the business cycle. Econometrica, 57, 357-384.

Hamilton, J. D. (1994). Time series analysis. Princeton, NJ: Princeton university press.

Haslbeck, J. M. B., Bringmann, L. F., \& Waldorp, L. (2018). How to estimate time-varying vector autoregressive models? a comparison of two methods. arXiv Preprint, 1711.05204.

Haslbeck, J. M. B., \& Waldorp, L. (2018). MGM: Structure estimation for time-varying mixed graphical models in highdimensional data. the journal of statistical software. arXiv Preprint, 1510.06871

Hastie, T. J., \& Tibshirani, R. J. (1990). Generalized additive models. Boca Raton, FL: Chapman and Hall/CRC.

Houben, M., Van Den Noortgate, W., \& Kuppens, P. (2015). The relation between short-term emotion dynamics and psychological well-being: A meta-analysis. Psychological bulletin, 141(4), 901-930.

Jahng, S., Wood, P. K., \& Trull, T. J. (2008). Analysis of affective instability in ecological momentary assessment: Indices using successive difference and group comparison via multilevel modeling. Psychological Methods, 13(4), 354-375.

Kossakowski, J., De Groot, P., Haslbeck, J., Borsboom, D., \& Wichers, M. (2017). Data from 'critical slowing down as a personalized early warning signal for depression'. Journal of Open Psychology Data, 5. doi: 10.5334/jopd.29

Koval, P., \& Kuppens, P. (2012). Changing emotion dynamics: Individual differences in the effect of anticipatory social stress on emotional inertia. Emotion, 12(2), 256-267.

Koval, P., Kuppens, P., Allen, N. B., \& Sheeber, L. (2012). Getting stuck in depression: The roles of rumination and emotional inertia. Cognition $\mathcal{E}$ emotion, 26(8), 1412-1427.

Koval, P., Pe, M. L., Meers, K., \& Kuppens, P. (2013). Affect dynamics in relation to depressive symptoms: Variable, unstable or inert? Emotion, 13(6), 1132.

Krone, T., Albers, C. J., Kuppens, P., \& Timmerman, M. E. (2017). A multivariate statistical model for emotion dynamics. , 18(5), 739-754.

Kuppens, P., Allen, N. B., \& Sheeber, L. B. (2010). Emotional inertia and psychological maladjustment. Psychological Science, 21, 984-991.

Kuppens, P., Champagne, D., \& Tuerlinckx, F. (2012). The dynamic interplay between appraisal and core affect in daily life. Frontiers in Psychology, 3, 1-8.

Kuppens, P., \& Verduyn, P. (2015). Looking at emotion regulation through the window of emotion dynamics. Psychological Inquiry, 26(1), 72-79.

Kuppens, P., \& Verduyn, P. (2017). Emotion dynamics. Current opinion in psychology, 17, 22-26.

Molenaar, P. C. M., De Gooijer, J. G., \& Schmitz, B. (1992). Dynamic factor analysis of nonstationary multivariate time series. Psychometrika, 57(3), 333-349.

Nelson, B., McGorry, P. D., Wichers, M., Wigman, J. T., \& Hartmann, J. A. (2017). Moving from static to dynamic models of the onset of mental disorder: a review. JAMA psychiatry, 74(5), 528-534.

Pe, M. L., Kircanski, K., Thompson, R. J., Bringmann, L. F., Tuerlinckx, F., Mestdagh, M., ... Gotlib, I. H. (2015). Emotion-network density in major depressive disorder. Clinical Psychological Science, 3(2), 292-300. doi: 10.1177/2167702614540645

Rovine, M. J., \& Walls, T. A. (2006). Multilevel autoregressive modeling of interindividual differences in the stability of a process. In T. A. Walls \& J. L. Schafer (Eds.), Models for 
intensive longitudinal data (pp. 124-147). Oxford, England: Oxford University Press.

Ryan, O., Kuiper, R. M., \& Hamaker, E. L. (2019). A continuoustime approach to intensive longitudinal data: What, why, and how? In Continuous time modeling in the behavioral and related sciences (pp. 27-54). Springer.

Scheffer, M., Bascompte, J., Brock, W. A., Brovkin, V., Carpenter, S. R., Dakos, V., ... Sugihara, G. (2009). Early-warning signals for critical transitions. Nature, 461(7260), 53-59.

Schuurman, N. K., Ferrer, E., de Boer-Sonnenschein, M., \& Hamaker, E. L. (2016). How to compare cross-lagged associations in a multilevel autoregressive model. Psychological Methods, 21(2), 206-261. doi: 10.1037/met0000062

Suls, J., Green, P., \& Hillis, S. (1998). Emotional reactivity to everyday problems, affective inertia, and neuroticism. Personality and Social Psychology Bulletin, 24(2), 127-136.

Suls, J., \& Martin, R. (2005). The daily life of the garden-variety neurotic: Reactivity, stressor exposure, mood spillover, and maladaptive coping. Journal of Personality, 73(6), 14851510.

Trull, T. J., \& Ebner-Priemer, U. (2013). Ambulatory assessment. Annual Review of Clinical Psychology, 9, 151-176.

Trull, T. J., Lane, S. P., Koval, P., \& Ebner-Priemer, U. W. (2015). Affective dynamics in psychopathology. Emotion Review, 7(4), 355-361.

van de Leemput, I. A., Wichers, M., Cramer, A. O., Borsboom, D., Tuerlinckx, F., Kuppens, P., ... Scheffer, M. (2014). Critical slowing down as early warning for the onset and termination of depression. Proceedings of the National Academy of Sciences, 111(1), 87-92.

Verduyn, P., Van Mechelen, I., Tuerlinckx, F., Meers, K., \&
Van Coillie, H. (2009). Intensity profiles of emotional experience over time. Cognition and Emotion, 23(7), 14271443.

Voelkle, M. C., \& Oud, J. H. (2013). Continuous time modelling with individually varying time intervals for oscillating and non-oscillating processes. British Journal of Mathematical and Statistical Psychology, 66(1), 103-126.

Walls, T. A., \& Schafer, J. L. (2006). Models for intensive longitudinal data. Oxford, England: Oxford University Press.

Wenze, S. J., Gunthert, K. C., Forand, N. R., \& Laurenceau, J.-P. (2009). The influence of dysphoria on reactivity to naturalistic fluctuations in anger. Journal of Personality, 77(3), $795-824$.

Wichers, M., Groot, P. C., Psychosystems, ESM Group, \& EWS Group. (2016). Critical slowing down as a personalized early warning signal for depression. Psychotherapy and Psychosomatics, 85(2), 114-116. doi: 10.1159/000441458

Wichers, M., Schreuder, M. J., Goekoop, R., \& Groen, R. N. (2019). Can we predict the direction of sudden shifts in symptoms? transdiagnostic implications from a complex systems perspective on psychopathology. Psychological medicine, 49(3), 380-387.

Wigman, J., van Os, J., Borsboom, D., Wardenaar, K., Epskamp, S., Klippel, A., ... Wichers, M. (2015). Exploring the underlying structure of mental disorders: Crossdiagnostic differences and similarities from a network perspective using both a top-down and a bottom-up approach. Psychological Medicine, 45(11), 2375-2387. doi: 10.1017/S0033291715000331

Wood, S. N. (2006). Generalized additive models: An introduction with R. Boca Raton, FL: Chapman and Hall/CRC. 


\title{
Supplemental Material to 'Inspecting gradual and abrupt changes in emotion dynamics with the time-varying change point autoregressive model'
}

\author{
Casper J. Albers* and Laura F. Bringmann* \\ * Joint first author \\ University of Groningen
}

\section{Introduction}

This is the supplementary material for the main article, which introduces the time-varying change point autoregressive model (TVCP-AR). This model extends the standard AR(1) model to allow for both smooth and sudden changes over time in the parameters of the model. For a full motivation of the model, the reader is referred to main text. Here we provide additional technical details, including an extensive simulation study. To keep this supplementary material self-containing, the notation of the model is shortly repeated here. Furthermore, references to related technical literature are provided.

The TVCP-AR model for time series $y_{1}, \ldots, y_{T}$ combines the AR(1) model with elements from the TV-AR model (Bringmann et al., 2017; Dahlhaus, 1997) and the change point model (Hamilton, 1989). The TV-AR model (see Bringmann et al., 2017, for technical details) allows for gradual changes in dynamics and the change point part deals with sudden changes. The version of the change point model we employ follows the ideas by Hamilton $(1989,1994)$, who provides

$$
y_{t}= \begin{cases}\beta_{0}+\beta_{1} y_{t-1}+\varepsilon_{t} & s_{t}=0 \\ \beta_{0}+\delta+\beta_{1} y_{t-1}+\varepsilon_{t} & s_{t}=1 .\end{cases}
$$

Thus, whenever $s_{t}=0$, the intercept of this model is $\beta_{0}$, and whenever $s_{t}=1$ it is $\beta_{0}+\delta$. In Hamilton's formulation the regimes are only different with respect to the value of the intercept, but it is straightforward to extend this to also allow for differing autoregression coefficients.

Heymans Institute for Psychological Research, University of Groningen, Grote Kruisstraat 2/1, 9712 TS Groningen, The Netherlands. Contact: c.j.albers@ rug.nl.

We thank the editor and two anonymous reviewers for their input and Markus Eronen for helpful comments on earlier versions of the manuscript. The Center for Information Technology of the University of Groningen provided access to the Peregrine high performance computing cluster.

Laura Bringmann's research was supported by the Netherlands Organization for Scientific Research (NWO; Veni grant 191G.037).
The process $s_{t} \in\{0,1\}, t=1, \ldots, T$ defines how the model behaves. When the values $s_{t}$ are independent Bernoulli realizations, one obtains the random switching model (Quandt, 1972). When $s_{t}$ is depending on the data, for example, $s_{t}=1$ if and only if $y_{t-1} \leq c$ for some (known) threshold $c$, the model is called a threshold model. The single structural change point model is obtained when $s_{t}=0$ for $t \leq \tau$ and $s_{t}=1$ for $t>\tau$ where $\tau$ is estimated from the data. It is possible to extend this model to include multiple change points, as we will show later.

Hamilton's model is the basis for a broad class of models. Besides change point models, this includes the CUSUM model (Page, 1954), Krylov subspace learning models (Idé \& Tsuda, 2007), Bayesian approaches (e.g. Pollak \& Siegmund, 1991) and various other modelling techniques (see Basseville \& Nikiforov, 1993, for a detailed discussion). Although these models have their merits, they are not based on the AR(1) framework and therefore not easy to combine with time-varying models.

The abrupt change can occur at a known moment, such as the moment where the medication dosage is altered. The confirmatory TVCP-AR model, combining the TV-AR model with the change point model, is developed for such situations. It is, however, also possible that the abrupt change happens at an unknown moment (also in hindsight). This is common in emotion research, as there are many factors influencing someone's wellbeing and it is oftentimes not feasible to attribute a change to a certain event. For these situations, the exploratory TVCP-AR model is needed. This model combines the TV-AR model with the structural change model.

\section{Confirmatory TVCP-AR models}

The confirmatory TVCP-AR model is surprisingly simple, combining the ideas of time-varying parameters (Bringmann et al., 2017) and of sudden changes (Hamilton, 1989). With a change point at time point $I$, the TVCP-AR model reads

$$
y_{t}=\left\{\begin{array}{cc}
\beta_{0, t}^{(0)}+\beta_{1, t}^{(0)} y_{t-1}+\varepsilon_{t} . & \text { if } t \leq I \\
\beta_{0, t}^{(0)}+\beta_{0, t}^{(1)}+\left(\beta_{1, t}^{(0)}+\beta_{1, t}^{(1)}\right) y_{t-1}+\varepsilon_{t} & \text { if } t>I
\end{array}\right.
$$


Here, the only difference with Hamilton's model is that all regression coefficients are allowed to change gradually over time, using the TV-AR framework, whereas in Hamilton's model these coefficients were fixed.

Using code variable

$$
D_{t}= \begin{cases}0 & t \leq I \\ 1 & t>I\end{cases}
$$

the TVCP-AR model can be rewritten as

$y_{t}=\beta_{0, t}^{(0)}+\left(\beta_{0, t}^{(1)}-\beta_{0, t}^{(0)}\right) D_{t}+\beta_{1, t}^{(0)} y_{t-1}+\left(\beta_{1, t}^{(1)}-\beta_{1, t}^{(0)}\right) D_{t} \times y_{t-1}+\varepsilon_{t}$.

By redefining the notation for the regression coefficients, the model simplifies to

$$
y_{t}=\beta_{0, t}^{(0)}+\beta_{0}^{(1)} D_{t}+\beta_{1, t}^{(0)} y_{t-1}+\beta_{1}^{(1)} D_{t} \times y_{t-1}+\varepsilon_{t} .
$$

In this model, the intercept $\beta_{0}^{(1)}$ and autoregression $\beta_{1}^{(1)}$ corresponding to the dummy-variable $D$ need not be time-varying, as the dummy variable can only attain two values. The TVAR model is the special case of this model when $\beta_{0}^{(1)} \equiv \beta_{1}^{(1)} \equiv$ 0 . Note that the model in (2) is the TVCP-AR(1) model. It is straightforward to extend this model to higher lag orders, if desired, or to rewrite the model such that a sudden change can only appear in either the intercept or the autoregression variable, but not both. In Hamilton's static model, the Chow test (Chow, 1960) was used to test for significance of the structural break. Testing for significance of $\beta_{0}^{(1)}$ and $\beta_{1}^{(1)}$ in (2) is straightforward within the GAM-model. By comparing the AIC, BIC, or other fit measure of model (2) with and without the restriction that $\beta_{0}^{(1)}=\beta_{1}^{(1)}=0$, one can assess the impact of $\beta_{0}^{(1)}$ and $\beta_{1}^{(1)}$ simultaneously.

\section{Exploratory TVCP-AR models}

There are many situations where it is unknown whether there is a change point and, if so, where this point lies. Now the challenge is two-fold: (i) finding out when the change takes place, (ii) finding out how large the sudden change in dynamics is. For the second question, we can directly apply the confirmatory approach mentioned above. For the first question, we outline our approach below. The approach is similar to that behind Quandt's Likelihood-Ratio Test (Quandt, 1960) and Hamilton's approach (Hamilton, 1989).

However, whereas the mathematical derivations of test bounds are possible for the AR(1) model with change points (Andrews, 1993), this is not the case for the more sophisticated time varying alternative discussed in this paper. That is why, in the next section, we will use an extensive simulation study to show that this approach works.

Without knowledge of where the change takes place, one should check all possible options. A change point model partitions the data into two periods: the one up to the switch and the one after the switch. A switch at time point 1,2, $T-1$ or $T$ is not possible, as this would imply that one of the partitions only has 0 or 1 measurements.

Also change points at time points close the edges of the time interval, e.g. at $t=4$, are undesired as this yields a partition with a time length that is too small to obtain robust estimates. Furthermore, abrupt changes that occur too close to the boundaries of the interval or to each other are difficult to detect. Thus, how many time points $k$ away from the boundaries of the interval or other change points one wants to stay depends on the context.

Our exploratory TVCP-AR algorithm is as follows. First, fit a TV-AR model without change points and denote the corresponding AIC-value by $\mathrm{AIC}^{0}$.

Second, for each $k<i<T-k+1$, perform the following actions:

1. Set up a dummy variable $D_{i}$ with value 0 for the first $i$ values and value 1 for the $T-i$ remaining values;

2. Fit the model

$$
y_{t}=\beta_{0, t}^{(0)}+\beta_{0}^{(1)} D_{i}+\beta_{1, t}^{(0)} y_{t-1}+\beta_{1}^{(1)} D_{i} \times y_{t-1}+\varepsilon_{t}
$$

to the data.

3. Denote the AIC-value of this model by $\mathrm{AIC}_{i}^{1}$.

Let $\mathrm{AIC}^{1}=\operatorname{argmin}_{i} \mathrm{AIC}_{i}^{1}$ where $j$ is the value for which this minimum is attained. Compute $\mathrm{AIC}^{1}-\mathrm{AIC}^{0}$. If this value is above a certain threshold, say -5 , then the difference in AIC is too small to denote $j$, a location with change point. If, however, the value does lie below this threshold, the difference is sufficient and location $j$ will be denoted as the first switch point. Note that besides the Akaike Information Criterion (AIC), any other information criterion, such as the $\mathrm{BIC}$, can be applied just as well. In the simulation section we will study both selection based on AIC and on BIC.

Once a change point has been found, the time series is partitioned into two parts. For the parts with sufficient remaining time points, the strategy above can be applied again to find a new change point. This approach can be repeated until either no further change points are detected or none of the partitions contains enough time points to sensibly apply the model.

\section{Simulation study}

To check the conditions in which our model is most useful we have set up an elaborative simulation study. In this simulation study, we varied the number of time points, the pattern of smooth variation for $\beta_{0}$ and $\beta_{1}$, and the size and location of change points. The simulations were performed at a high-performance computer cluster with 24 cores (Intel Xeon $2.5 \mathrm{GHz}$ ) running in parallel with $120 \mathrm{~GB}$ of memory.

\section{Simulation set-up}

The following settings are varied in the simulation design: 
- The time series length $T: 30,60,100,200,400,600$, 1000.

- The pattern of variation. We simulated 9 different conditions. Figure 1 shows each of the conditions. The first condition corresponds to a non-time-varying $\mathrm{AR}(1)$ model. Conditions $\mathrm{B}$ and $\mathrm{C}$ are smoothly varying, but without change point. In conditions $\mathrm{D}, \mathrm{E}$ and $\mathrm{F}$ there is no smooth variation but there is a change point (at three different locations). Condition $\mathrm{G}$ combines a smooth variation with a change point. In conditions $\mathrm{H}$ and I the change point manifests itself in either $\beta_{0}$ or $\beta_{1}$. We expect that abrupt changes in $\beta_{0}$ are easier to detect than those in $\beta_{1}$. Abrupt changes are easiest to detect when they occur in both $\beta_{0}$ and $\beta_{1}$ simultaneously, such as in conditions D - G. Note also that the conditions where there is no smooth variation but only a change point (e.g., conditions D, E and F) are more difficult to detect than conditions where there are abrupt and smooth or gradual changes (e.g., conditions $\mathrm{C}$ and $\mathrm{G}$ ). This is because the gradual part of the TVCP-AR model, the TV-AR model, is tailored for detecting (gradual) change.

- The size of the sudden change. We use two conditions, 'small' and 'large'. In the 'small' condition, a change point indicates an increase of $1 \mathrm{sd}$ in $\beta_{0}$ and of .3 in $\beta_{1}$. In the 'large' condition, a change point indicates an increase of $2 \mathrm{sd}$ in $\beta_{0}$ and of .7 in $\beta_{1}$.

This leads to a fully crossed $7 \times 9 \times 2$ design. For each cell, $R=300$ replications have been computed on basis of the AIC and another $R=300$ replications have been computed on basis of the BIC.

Model performance is measured through the following two metrics. The first metric measures how much better the model with change point at the correct switch point location is, compared to a model without a change point. This metric computes

$$
M=\frac{1}{R} \sum_{r=1}^{R}\left(\mathrm{AIC}_{j}^{(1)}-A I C^{(0)}\right)
$$

and

$$
S D=\sqrt{\frac{1}{R-1} \sum_{r=1}^{R}\left(\mathrm{AIC}_{j}^{(1)}-A I C^{(0)}-M\right)^{2}}
$$

with $j$ being the location where the change point takes place. (In conditions 1,2 and $3 j$ is set to $j=T / 2$ ). Thus, this metric studies whether the model (i) is capable of having an improved model fit when the correct change point is implemented in the model and, by reporting both mean and standard deviation, we can study the robustness of this result; (ii) leads to a worse model fit in conditions where there is no change point.
The second metric studies whether the change point is located at the right location. To this end, it computes

$$
\operatorname{median}|i-j|,
$$

with $j$ being the correct location of the change point, and $i$ the location with the lowest $\mathrm{AIC}_{i}^{(1)}$-value. This metric is only computed in conditions $4-9$ where there actually is a change point.

\section{Simulation results}

Before discussing the general results for all conditions, we focus on a specific cell in the fully crossed simulation design to explain the results in detail. This is Condition $\mathrm{D}$ (static $\mathrm{AR}(1)$ processes for both $\beta_{0}$ and $\beta_{1}$ with a single change point halfway the time period), with $T=200$ time points, change point at time 100 , and large changes at the change point. Detailed results of the replications for this condition are shown in Figure 2. From the left panel we learn that, on average, the AIC-difference is positive at time points $2, \ldots, 87$ and $109, \ldots, 199$, which indicates that the model with a change point at those time points, rightfully, fits worse than a model without change point. On the other hand, around, and not only exactly at time point 100, most replications clearly capture the change point. The average AICdifference at time 100 is $-20.080(s d=7.304)$. The right panel of Figure 2 displays smoothed histograms for each of the time points. For time points away from 100, this density peaks above zero. For the correct time point, 100, it is clearly much lower, with 281 out of 300 replications having an AIC-difference of -10 or less and 299 replications having a negative AIC-difference.

The figure also shows that for time points close to, but not equal to, 100, the AIC-difference is also predominantly negative. At time point 105, for example, 166 out of 300 replications provide a negative AIC-difference, with 28 replications scoring -5 or less and 6 replications scoring -10 or less.

How the estimation works out in practice is shown in Figure 3 for two out of the 300 replications. For instance, it shows that for the vast majority of points the true value is captured by the $95 \%$-confidence bands. It also indicates that the mean is easier to correctly estimate than the autoregressive effect.

Figure 5 shows results for conditions differing from the condition in Figure 2 in one respect: either (a) small changes at the change point, (b) a shorter time series, or (c) a condition with only smooth variation. Panels (a) and (b) show that, as expected, it is more difficult to detect change points when the changes at the switch are small (mean AIC-difference at $T / 2$ of -5.394 , sd $=4.867$ ) or when fewer time points are observed (mean -5.108 , sd 7.089). The third panel shows that in absence of any change point, the model will not structurally provide false positives. Figures 5 and 2 focus on Con- 
Condition A
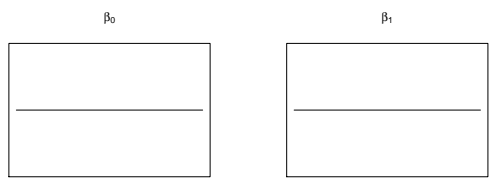

Condition D
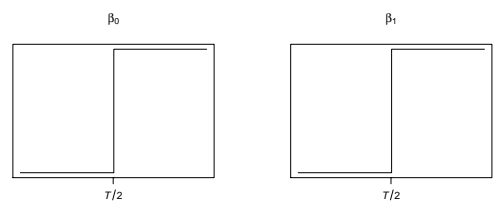

Condition G

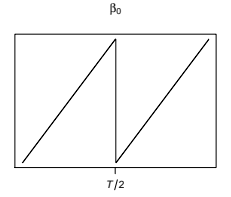

Condition B

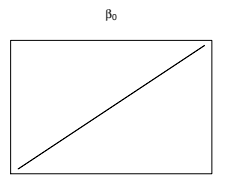

Condition E

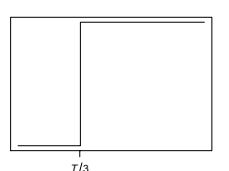

Condition $\mathrm{H}$

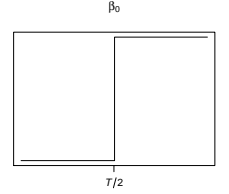

Condition C
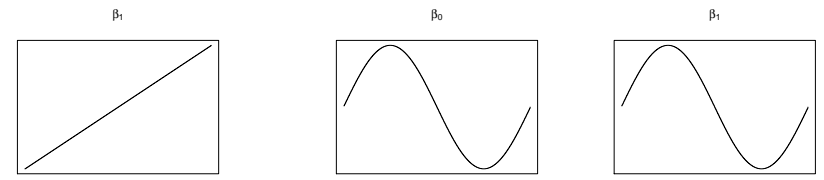

Condition F
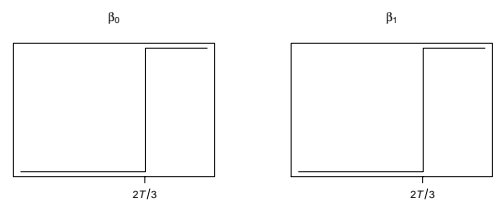

Condition I
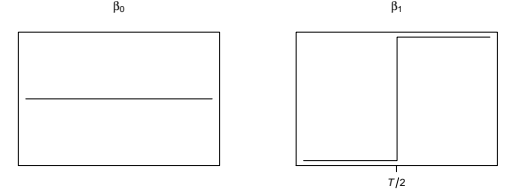

Figure 1. The nine conditions, A to I, for $\beta_{0}$ (intercept, left panels) and $\beta_{1}$ (autoregression parameter, right panel).

dition D. In Figure 4 details are given for two instances of Condition $\mathrm{G}$ for longer time series $(T=400)$.

The results for the first metric, mean and standard deviation of difference in AIC-score, are provided in Tables 1, for small change points, and 2, for large change points. The second metric, the median absolute difference, is given in Table 3 for the situation where selection is done based on AIC. Tables 4, 5, 6 report the same information, now when selection is done based on the BIC.

Table 1 summarizes the results when the changes in dynamics at the change point are small: $\beta_{0}$ increases by $1 \mathrm{sd}$ and $\beta_{1}$ by 0.3 . It can be seen that the conditions without change point (conditions A, B, and $\mathrm{C}$ ) on average have a higher AICvalue when a change point is included in the model. Thus, the model correctly penalizes the inclusion of unnecessary variables.

For conditions D, E, F, and G, all with one change point halfway the time series, the results are very similar. With $T=100$, the average AIC-difference is around 2.5, with $T=400$ it is around 10, and with $T=1000$ it is more than 20. There are various rules of thumb about when to accept a (negative) difference in AIC as substantial, ranging from 2 to 10 points (Burnham \& Anderson, 2002, Chapter 2).

Alternatively, one could perform model selection based on Akaike weights (Burnham \& Anderson, 2002; Wagenmakers $\&$ Farrel, 2004). If two models have an AIC-difference of $\Delta$, then the model with the lower AIC has a likelihood which is a factor $\exp (-\Delta / 2)$ higher than the one with the higher AIC. Thus, when comparing a model with change point with a model without switch, an AIC-difference of 2, 5 and 10 would imply that the one model is $2.7,12.2$, and 148 times as likely, respectively, as the other. However, given that almost $T$ different (yet related) models are being compared, some kind of multiple comparison correction needs to be applied, and this correction needs to be stronger for longer time series.

With a lenient rules of thumb of accepting 2 points difference in AIC as substantial, $T=100$ would be sufficient to spot a change point with a small change in dynamics, on average. However, the standard deviations are substantial, so in many cases such a change point will be missed. Furthermore, with a too lenient rule of thumb, the results for Conditions $\mathrm{A}, \mathrm{B}$, and $\mathrm{C}$ indicate that it will not be uncommon to have a 'false positive', i.e. to incorrectly indicate that a change point has taken place. This can be avoided by increasing the minimum difference in AIC required, at the cost of needing a larger sample size in order to have sufficient power.

This is not an issue when the changes in dynamics are large, such as 2 sd change for $\beta_{0}$ and 0.7 change for $\beta_{1}$. From Table 2 it can be seen that even with short time series of length $T=60$ the difference in AIC is substantial. Interestingly, Condition $\mathrm{G}$ now clearly outperforms the other models, something which was not the case with small changes.

From both Table 1 and 2, we can see that conditions $\mathrm{H}$ and I show that if the change point happens closer to either the beginning or the end of the time series, it is harder to detect: in the ideal situation both the 'before' and 'after' regimes have the same sample size. This ties in with the well-known statistical phenomenon that balanced designs are more powerful 

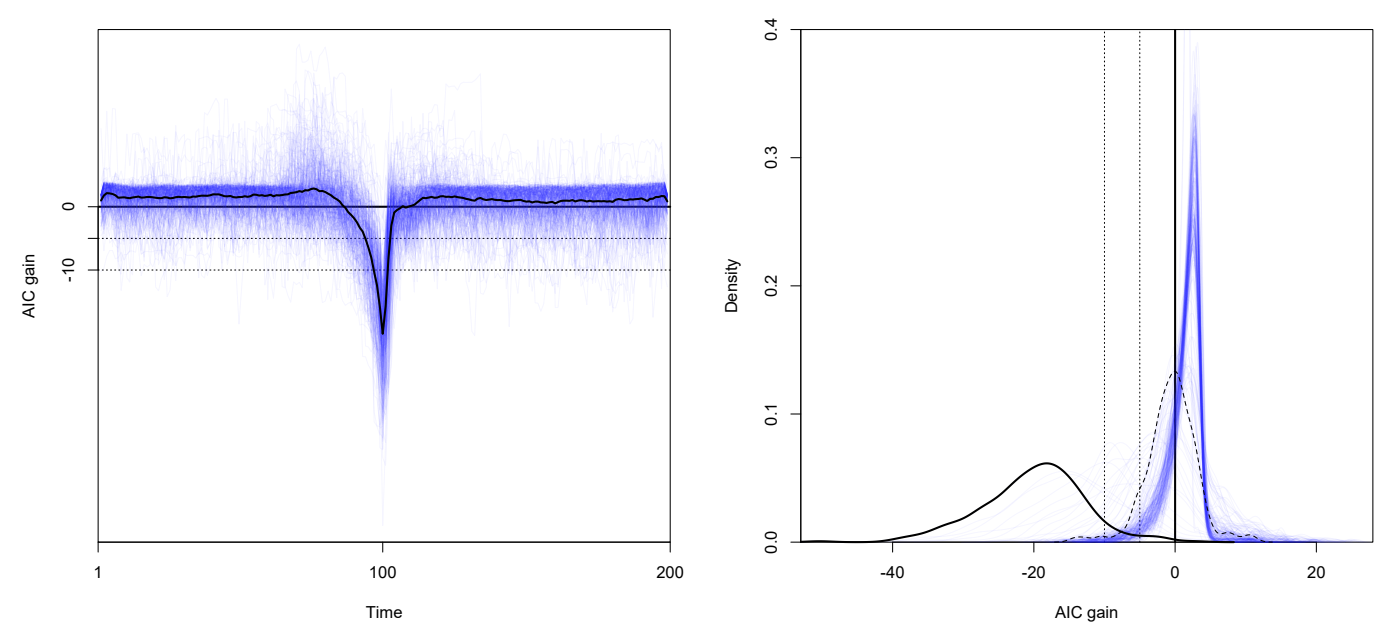

Figure 2. Detailed simulation results for the case with Condition D, large changes at the change point and $T=200$. Left panel: for each of the 300 replications the 'AIC difference'-curve is plotted by a thin blue line. The average of the 300 replications is displayed with a thick black line. Right panel: for each of the time point $(2, \ldots, 199)$ the density of AIC-difference over replications is displayed by a thin blue curve. The densities at $t=100$ (solid black) and $t=105$ (dashed black curve) have been highlighted.

than unbalanced designs.

Table 3 looks at the median absolute difference (MAD) between the actual location of the change point, and the location $i$ where $\mathrm{AIC}_{i}^{(1)}$ is smallest. This number is obviously related to the length of the time series. From the table we can observe that when the changes at the change point are small, $T>100$ is required for having an acceptable MAD. When the changes at the change point are large, $T>60$ suffices. The MAD is minimal, even for small changes, for time series lengths of $T>200$. A MAD-value of 1 indicates that for more than 50 percent of replications, the assigned change point was either fully correct or, at most, one time point off. It should be noted that even in these best conditions, the simulation sometimes gives a very wrong inference. In most conditions, a few out of the 300 replications find the smallest $\mathrm{AIC}_{i}^{(1)}$ value at a time point below 10 or above $T-10$.

Tables 4 and 5 display the results of the first metric when the Bayesian Information Criterion (BIC) is used. In general, this approach yields comparable findings as the one based on the Akaike Information Criterion. This is not surprising, as in the exploratory TVCP-AR search to the optimal location of an abrupt change, the AIC and BIC-based approaches will find the same location. They can differ in whether the difference in AIC/BIC-score is sufficiently substantial or not. The BIC-approach will require other thresholds, as the $M$-values based on the BIC seem to be roughly twice those based on the AIC. Based on the median absolute differences (Table 6 ), it seems that selection based on BIC slightly outperforms that based on AIC, in terms of median absolute difference, especially for shorter time series lengths. Even with $T=100$ already acceptable results can be obtained for small differences at the change point.

In practice, one has to decide upon a threshold for when to call the $\mathrm{AIC}^{0}-\mathrm{AIC}^{1}$ change sufficient to implement it in the model. In the empirical example in Albers and Bringmann (2020), this threshold is set to -10 . Changing the threshold changes the trade-off between false positives (flagging abrupt changes at locations without change) and false negatives (failure to find actual changes).

Table 7 studies the false positive rate, for Conditions A, $\mathrm{B}$ and $\mathrm{C}$. Besides the threshold choice -10 , also -15 and -5 are studied. For each cell in the table, it is studied for how many of the 300 replications there was a $\mathrm{AIC}^{0}-\mathrm{AIC}^{1}$-value below the threshold. As the model can be unstable close to the edges of the time series, the first and last ten values of $\mathrm{AIC}^{0}-\mathrm{AIC}^{1}$ are omitted.

The AIC-based results suggest that a threshold of -10 yields a false positive rate somewhat above the usual $\alpha=.05$ but a threshold of -15 clearly is too strict. For the BIC, -15 seems to be more appropriate as threshold.

In a similar fashion the true positive rate, for conditions D-I with an abrupt change, is computed. Here, it is checked at what rate changes are correctly identified. As identifications on time points close to, but not exactly equal to, the true moment of change are acceptable, all identified changes within 5 time points of the true change are counted as true positives. The results in Tables $8(T<400)$ and $9(T \geq 400)$ show that, for power considerations, using -10 as threshold clearly is preferred over using -15 . Furthermore, the BICselection tool yields better power and for short time series 

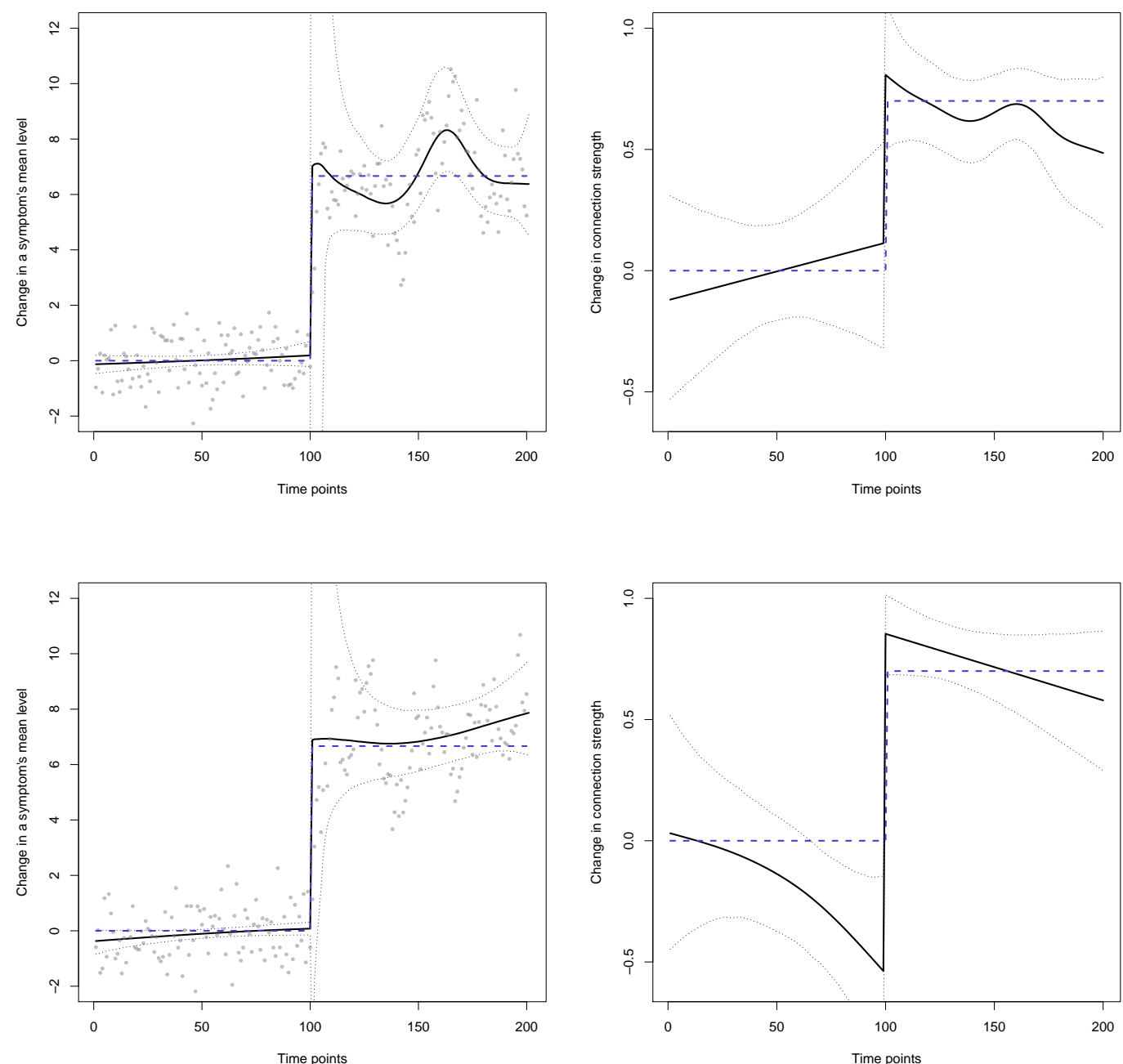

Figure 3. Detailed simulation results for two instances of Condition D with large changes at the change point and $T=200$. The top row and bottom row are separate instances. The left column displays the simulated data (dots), the resulting $\mu$-level (dashed line), and the resulting estimated $\mu$-level (thick solid line) plus $95 \%$ credible bands (dotted). The column on the right displays the results for the inertia $\beta_{1}$.

the model clearly is underpowered.

\section{Technical conclusions}

It is important to stress that the separate components for modeling gradual change and abrupt change are not new, but based on results from literature that have proven to be successful in modeling psychological time series. The combination of both methods into a single model, however, is new. What is especially new, is the detailed simulation study to investigate the performance of this model under a wide range of circumstances that are common in psychological studies with experience sampling data.

In the simulation study we have shown that our model is equipped for typical psychological data sets: for time se- ries of length $T=100$ it has good power to detect large abrupt changes and reasonable power to detect smaller abrupt changes. It should be stressed that for smaller time series (e.g., $T<50$ ), the model will be underpowered. However, as time series are increasing in length due to advances in data collection techniques, situations with sufficient data for our model will become more and more abundant.

The TV-AR model has been extended to the multivariate case (i.e., modelling multiple emotions simultaneously) by Bringmann, Ferrer, Hamaker, Borsboom, and Tuerlinckx (2018), who denoted it the TV-VAR model. An alternative multivariate time-varying model is provided by Haslbeck and Waldorp (2018). A comparison of both methods (Haslbeck, Bringmann, \& Waldorp, 2018) highlights that both ap- 

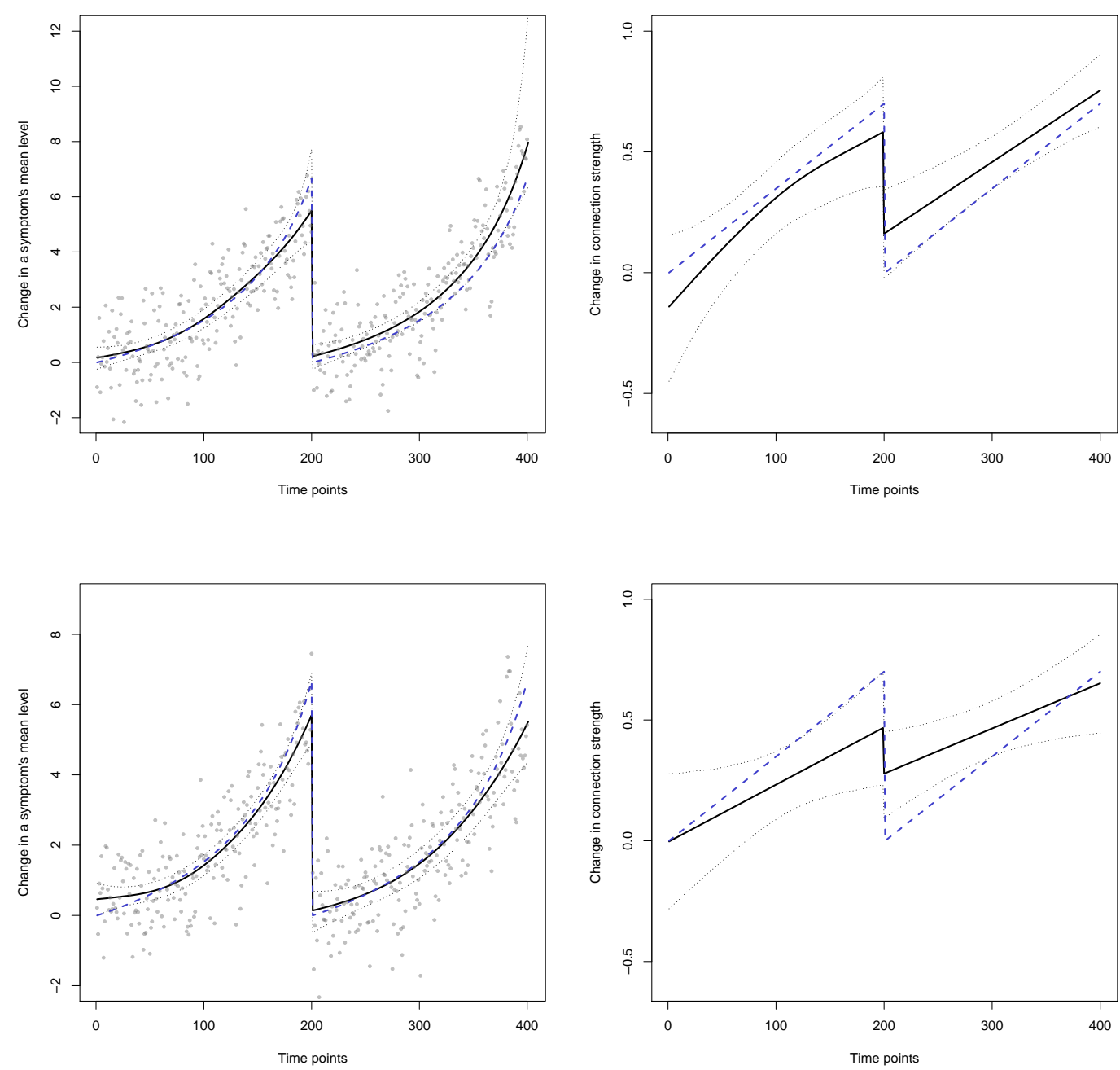

Figure 4. Similar figure as Figure 3, but now with $T=400$ and Condition G.

(a)

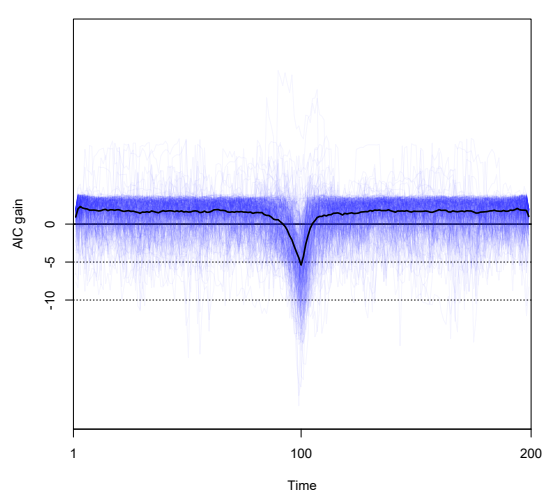

(b)

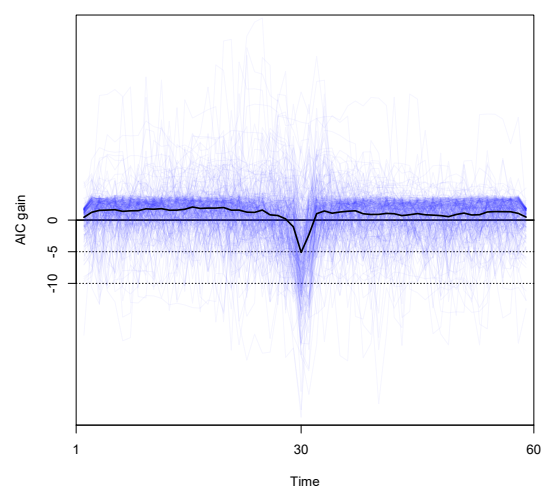

(c)

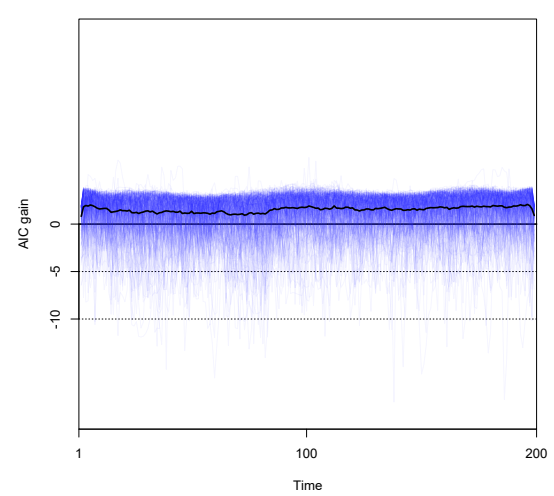

Figure 5. Similar visualisation as in the left panel of Figure 2, now for three situations that differ at one time point from the situation in Figure 2: (a) Condition D, 200 time points, small changes at the change point; (b) Condition D, 60 time points, large changes at the change point; (c) Condition C, 200 time points, gradual change without a change point. 
proaches have good performance. In theory, this model can directly be combined with the change point model in the same way we did in this paper for the TV-AR model: find time point $i$ such that the sum of the AICs or BICs of the TV-VAR applied to the data before $i$ and the TV-VAR applied to the data from $i$ onwards is minimal. As the number of cross-lagged correlations grows quadratically with the number of variables involved, it can be assumed that larger time series are necessary to have sufficient power for finding abrupt changes. Future simulations are necessary to study this in further detail.

In our work, we do not attempt to give definitive answers to the question what the minimum length of a time series should be. Furthermore, our paper does not give a definitive answer to the question what difference in AIC or BIC should be accepted as being substantial enough to claim the existence of a change point, although Tables 7, 8 and 9 provide some initial information. This is intentional and in line with the strong advice from Burnham and Anderson (2002) against such general rules of thumb. Similar to a power analysis, there are multiple facets at play simultaneously here. The objective of setting the value of threshold should be such that true change points will be detected as well as possible ('high power'), with as few as possible false positives ('Type I errors'). The value of the threshold should, at least, depend on the length of the time series and on the effect size at the change point one wants or expects to find.

\section{References}

Albers, C. J., \& Bringmann, L. F. (2020). Inspecting gradual and abrupt changes in emotion dynamics with the time-varying change point autoregressive model. European Journal for Psychological Assessment, accepted for publication.

Andrews, D. W. K. (1993). Tests for parameter instability and structural change with unknown change point. Econometrica, 61, 821-856. doi: $10.2307 / 2951764$

Basseville, M., \& Nikiforov, I. V. (1993). Detection of abrupt changes - theory and application. Englewood Cliffs, NJ: Prentice Hall.

Bringmann, L. F., Ferrer, E., Hamaker, E. L., Borsboom, D., \& Tuerlinckx, F. (2018). Modeling nonstationary emotion dy- namics in dyads using a time-varying vector-autoregressive model. Multivariate behavioral research, 53(3), 293-314.

Bringmann, L. F., Hamaker, E. L., Vigo, D. E., Aubert, A., Borsboom, D., \& Tuerlinckx, F. (2017). Changing dynamics: Time-varying autoregressive models using generalized additive modeling. Psychological Methods, 22(3), 409-425. doi: 10.1037/met0000085

Burnham, K., \& Anderson, D. (2002). Model selection and multimodel inference (second ed.). New York: Springer.

Chow, G. C. (1960). Tests of equality between sets of coefficients in two linear regressions. Econometrica, 28, 591605. doi: $10.2307 / 1910133$

Dahlhaus, R. (1997). Fitting time series models to nonstationary processes. The Annals of Statistics, 25(1), 1-37.

Hamilton, J. D. (1989). A new approach to the economic analysis of nonstationary time series and the business cycle. Econometrica, 57, 357-384.

Hamilton, J. D. (1994). Time series analysis. Princeton, NJ: Princeton university press.

Haslbeck, J. M. B., Bringmann, L. F., \& Waldorp, L. (2018). How to estimate time-varying vector autoregressive models? a comparison of two methods. arXiv Preprint, 1711.05204.

Haslbeck, J. M. B., \& Waldorp, L. (2018). MGM: Structure estimation for time-varying mixed graphical models in highdimensional data. the journal of statistical software. arXiv Preprint, 1510.06871.

Idé, T., \& Tsuda, K. (2007). Change-point detection using krylov subspace learning. In Proceedings of the 2007 siam international conference on data mining (p. 515-520). doi: $10.1137 / 1.9781611972771 .54$

Page, E. S. (1954). Continuous inspection scheme. Biometrika, 41, 100-115. doi: 10.1093/biomet/41.1-2.100

Pollak, M., \& Siegmund, D. (1991). Sequential decision of a change in a normal mean when the initial value is unknown. Annals of Statistics, 19, 394-416.

Quandt, R. E. (1960). Tests of the hypothesis that a linear regression obeys two separate regimes. Journal of the American Statistical Association, 55, 32430. doi: 10.2307/2281745

Quandt, R. E. (1972). A new approach to estimating switching regressions. Journal of the American Statistical Association, $67,306310$.

Wagenmakers, E.-J., \& Farrel, S. (2004). AIC model selection using Akaike weights. Psychonomic Bulletin $\mathcal{E}$ Review, 11(1), 192-196. 
Table 3

\begin{tabular}{|c|c|c|c|c|c|c|c|c|c|c|c|c|c|c|}
\hline \multirow[b]{2}{*}{ Cond. } & \multicolumn{2}{|c|}{$T=30$} & \multicolumn{2}{|c|}{$T=60$} & \multicolumn{2}{|c|}{$T=100$} & \multicolumn{2}{|c|}{$T=200$} & \multicolumn{2}{|c|}{$T=400$} & \multicolumn{2}{|c|}{$T=600$} & \multicolumn{2}{|c|}{$T=1000$} \\
\hline & $\mathrm{S}$ & $\mathrm{L}$ & $S$ & $\mathrm{~L}$ & $S$ & $\mathrm{~L}$ & $\mathrm{~S}$ & $\mathrm{~L}$ & $\mathrm{~S}$ & $\mathrm{~L}$ & $S$ & $\mathrm{~L}$ & $S$ & $\mathrm{~L}$ \\
\hline $\mathrm{D}$ & 6.0 & 6.0 & 14.0 & 5.0 & 14.5 & 1.0 & 3.0 & 1.0 & 2.0 & 1.0 & 2.0 & 1.0 & 1.0 & 1.0 \\
\hline $\mathrm{E}$ & 6.0 & 6.0 & 12.0 & 10.0 & 13.0 & 1.0 & 5.0 & 1.0 & 2.0 & 1.0 & 2.0 & 1.0 & 2.0 & 1.0 \\
\hline $\mathrm{F}$ & 6.0 & 7.0 & 12.0 & 4.0 & 14.0 & 1.0 & 3.5 & 1.0 & 2.0 & 1.0 & 2.0 & 1.0 & 1.0 & 1.0 \\
\hline $\mathrm{G}$ & 6.0 & 1.0 & 10.0 & 1.0 & 8.0 & 1.0 & 3.0 & 1.0 & 1.5 & 1.0 & 1.0 & 1.0 & 1.0 & 1.0 \\
\hline $\mathrm{H}$ & 6.0 & 6.0 & 12.0 & 11.0 & 18.0 & 9.0 & 13.0 & 1.0 & 3.0 & 1.0 & 3.0 & 1.0 & 2.0 & 1.0 \\
\hline I & 6.0 & 7.0 & 14.0 & 11.0 & 25.0 & 2.0 & 49.0 & 1.0 & 99.0 & 1.0 & 99.0 & 1.0 & 47.5 & 1.0 \\
\hline
\end{tabular}

Median absolute difference median $|i-j|$ over the 300 replications, with $j$ being the correct location of the change point and $i$ being the location where $\mathrm{AIC}_{i}^{(1)}$ is lowest. Reported are results for small $(S)$ and large $(L)$ sudden changes. 

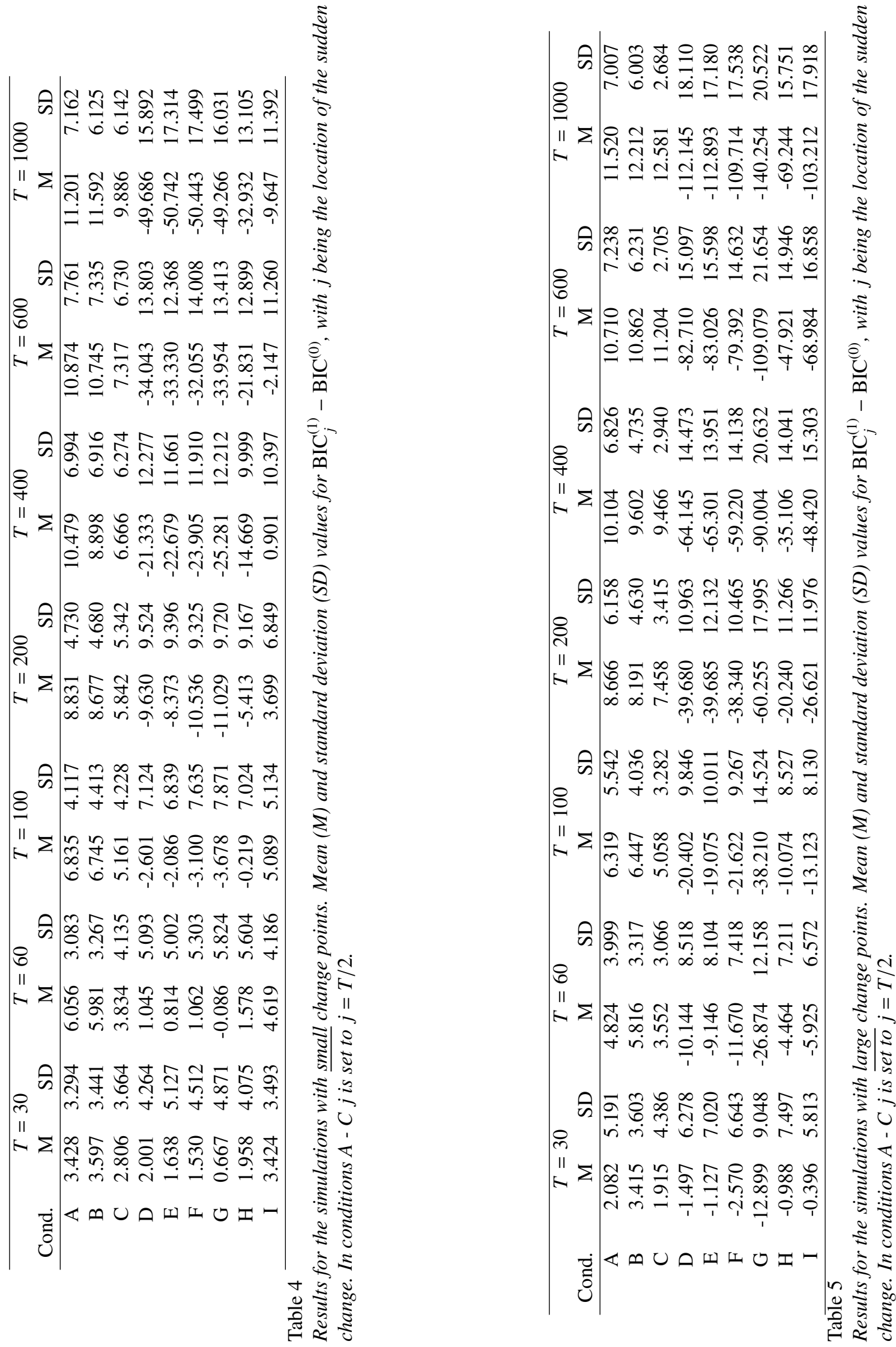


\begin{tabular}{rrrrrrrrrrrrrrr}
\hline & \multicolumn{4}{c}{$T=30$} & \multicolumn{1}{c}{$T=60$} & \multicolumn{2}{c}{$T=100$} & \multicolumn{2}{c}{$T=200$} & \multicolumn{2}{c}{$T=400$} & \multicolumn{2}{c}{$T=600$} & \multicolumn{2}{c}{$T=1000$} \\
Cond. & $\mathrm{S}$ & $\mathrm{L}$ & $\mathrm{S}$ & $\mathrm{L}$ & $\mathrm{S}$ & $\mathrm{L}$ & $\mathrm{S}$ & $\mathrm{L}$ & $\mathrm{S}$ & $\mathrm{L}$ & $\mathrm{S}$ & $\mathrm{L}$ & $\mathrm{S}$ & $\mathrm{L}$ \\
\hline $\mathrm{D}$ & 6.0 & 4.0 & 8.0 & 1.0 & 3.0 & 1.0 & 2.0 & 1.0 & 2.0 & 1.0 & 2.0 & 1.0 & 2.0 & 1.0 \\
$\mathrm{E}$ & 6.0 & 5.0 & 7.0 & 1.0 & 3.0 & 1.0 & 2.0 & 1.0 & 2.0 & 1.0 & 2.0 & 1.0 & 2.0 & 1.0 \\
$\mathrm{~F}$ & 5.0 & 4.0 & 7.0 & 1.0 & 2.0 & 1.0 & 2.0 & 1.0 & 2.0 & 1.0 & 2.0 & 1.0 & 2.0 & 1.0 \\
$\mathrm{G}$ & 5.0 & 1.0 & 5.0 & 1.0 & 2.0 & 1.0 & 1.0 & 1.0 & 1.0 & 1.0 & 1.0 & 1.0 & 2.0 & 1.0 \\
$\mathrm{H}$ & 6.0 & 5.0 & 10.0 & 2.0 & 7.0 & 1.0 & 3.0 & 1.0 & 2.0 & 1.0 & 2.0 & 1.0 & 2.0 & 1.0 \\
$\mathrm{I}$ & 7.0 & 5.0 & 17.0 & 2.0 & 25.5 & 1.0 & 42.5 & 1.0 & 48.0 & 1.0 & 20.0 & 1.0 & 13.0 & 1.0 \\
\hline
\end{tabular}

Table 6

Median absolute difference median $|i-j|$ over the 300 replications, with $j$ being the correct location of the change point and $i$ being the location where $\mathrm{BIC}_{i}^{(1)}$ is lowest. Reported are results for small $(S)$ and large $(L)$ sudden changes. 
Table 7

\begin{tabular}{|c|c|c|c|c|c|c|c|c|c|c|c|c|c|}
\hline \multirow[t]{3}{*}{$N$} & \multirow[t]{3}{*}{ Cond. } & \multicolumn{6}{|c|}{ Small changes } & \multicolumn{6}{|c|}{ Large changes } \\
\hline & & \multicolumn{3}{|c|}{ AIC threshold } & \multicolumn{3}{|c|}{ BIC threshold } & \multicolumn{3}{|c|}{ AIC threshold } & \multicolumn{3}{|c|}{ BIC threshold } \\
\hline & & -15 & -10 & -5 & -15 & -10 & -5 & -15 & -10 & -5 & -15 & -10 & -5 \\
\hline \multirow[t]{3}{*}{30} & $\mathrm{~A}$ & .11 & .22 & .39 & .02 & .06 & .18 & .21 & .37 & .62 & .08 & .18 & .41 \\
\hline & B & .08 & .15 & .35 & .01 & .05 & .16 & .14 & .25 & .49 & .04 & .11 & .24 \\
\hline & $\mathrm{C}$ & .06 & .17 & .37 & .03 & .06 & .17 & .13 & .26 & .51 & .07 & .11 & .31 \\
\hline \multirow[t]{3}{*}{60} & A & .03 & .11 & .40 & .00 & .03 & .10 & .12 & .34 & .73 & .01 & .10 & .36 \\
\hline & $\mathrm{B}$ & .01 & .06 & .34 & .00 & .02 & .09 & .04 & .14 & .47 & .00 & .03 & .18 \\
\hline & $\mathrm{C}$ & .02 & .06 & .40 & .02 & .06 & .23 & .03 & .15 & .59 & .01 & .05 & .28 \\
\hline \multirow[t]{3}{*}{100} & $\mathrm{~A}$ & .00 & .05 & .40 & .01 & .05 & .17 & .07 & .27 & .73 & .06 & .16 & .37 \\
\hline & B & .01 & .07 & .32 & .02 & .07 & .14 & .03 & .10 & .41 & .03 & .07 & .16 \\
\hline & $\mathrm{C}$ & .00 & .07 & .55 & .02 & .08 & .31 & .03 & .13 & .63 & .00 & .05 & .25 \\
\hline \multirow[t]{3}{*}{200} & A & .00 & .07 & .43 & .03 & .08 & .17 & .02 & .18 & .65 & .06 & .15 & .24 \\
\hline & B & .01 & .02 & .39 & .03 & .10 & .18 & .01 & .10 & .47 & .04 & .07 & .19 \\
\hline & $\mathrm{C}$ & .01 & .09 & .60 & .04 & .14 & .37 & .02 & .13 & .70 & .03 & .05 & .18 \\
\hline \multirow[t]{3}{*}{400} & A & .00 & .05 & .43 & .06 & .11 & .21 & .02 & .11 & .55 & .10 & .18 & .26 \\
\hline & B & .00 & .04 & .44 & .07 & .13 & .22 & .01 & .10 & .48 & .04 & .10 & .22 \\
\hline & $\mathrm{C}$ & .01 & .08 & .68 & .07 & .17 & .42 & .02 & .13 & .74 & .01 & .04 & .09 \\
\hline \multirow[t]{3}{*}{600} & A & .00 & .06 & .46 & .07 & .13 & .23 & .01 & .13 & .58 & .09 & .12 & .22 \\
\hline & B & .00 & .06 & .43 & .06 & .12 & .20 & .00 & .09 & .47 & .04 & .10 & .20 \\
\hline & $\mathrm{C}$ & .01 & .12 & .76 & .04 & .15 & .43 & .02 & .11 & .79 & .00 & .03 & .11 \\
\hline \multirow[t]{3}{*}{1000} & A & .00 & .07 & .50 & .05 & .09 & .21 & .01 & .11 & .55 & .10 & .14 & .20 \\
\hline & B & .00 & .06 & .47 & .06 & .11 & .20 & .01 & .07 & .46 & .06 & .07 & .11 \\
\hline & $\mathrm{C}$ & .01 & .11 & .76 & .04 & .10 & .29 & .01 & .18 & .85 & .00 & .02 & .10 \\
\hline
\end{tabular}

Empirical false positive rates, for three levels of the threshold. 
Table 8

\begin{tabular}{|c|c|c|c|c|c|c|c|c|c|c|c|c|c|}
\hline \multirow[t]{3}{*}{$N$} & \multirow[t]{3}{*}{ Cond. } & \multicolumn{6}{|c|}{ Small changes } & \multicolumn{6}{|c|}{ Large changes } \\
\hline & & \multicolumn{3}{|c|}{ AIC threshold } & \multicolumn{3}{|c|}{ BIC threshold } & \multicolumn{3}{|c|}{ AIC threshold } & \multicolumn{3}{|c|}{ BIC threshold } \\
\hline & & -15 & -10 & -5 & -15 & -10 & -5 & -15 & -10 & -5 & -15 & -10 & -5 \\
\hline \multirow[t]{6}{*}{30} & $\mathrm{D}$ & 0.13 & 0.25 & 0.46 & 0.03 & 0.08 & 0.24 & 0.22 & 0.38 & 0.64 & 0.12 & 0.24 & 0.53 \\
\hline & $\mathrm{E}$ & 0.10 & 0.24 & 0.50 & 0.02 & 0.06 & 0.22 & 0.24 & 0.44 & 0.71 & 0.12 & 0.27 & 0.57 \\
\hline & $\mathrm{F}$ & 0.12 & 0.25 & 0.49 & 0.04 & 0.09 & 0.29 & 0.14 & 0.29 & 0.56 & 0.07 & 0.17 & 0.54 \\
\hline & $\mathrm{G}$ & 0.12 & 0.24 & 0.52 & 0.04 & 0.10 & 0.28 & 0.48 & 0.73 & 0.89 & 0.48 & 0.68 & 0.86 \\
\hline & $\mathrm{H}$ & 0.12 & 0.24 & 0.54 & 0.04 & 0.10 & 0.27 & 0.28 & 0.43 & 0.71 & 0.16 & 0.28 & 0.55 \\
\hline & I & 0.11 & 0.23 & 0.46 & 0.02 & 0.07 & 0.20 & 0.20 & 0.32 & 0.59 & 0.10 & 0.23 & 0.43 \\
\hline \multirow[t]{6}{*}{60} & $\mathrm{D}$ & 0.02 & 0.06 & 0.26 & 0.01 & 0.08 & 0.23 & 0.10 & 0.35 & 0.69 & 0.30 & 0.58 & 0.81 \\
\hline & $\mathrm{E}$ & 0.03 & 0.07 & 0.29 & 0.02 & 0.05 & 0.24 & 0.13 & 0.33 & 0.63 & 0.29 & 0.52 & 0.77 \\
\hline & $\mathrm{F}$ & 0.01 & 0.06 & 0.29 & 0.01 & 0.07 & 0.25 & 0.11 & 0.35 & 0.72 & 0.39 & 0.66 & 0.88 \\
\hline & $\mathrm{G}$ & 0.01 & 0.09 & 0.37 & 0.01 & 0.09 & 0.30 & 0.72 & 0.84 & 0.97 & 0.83 & 0.92 & 0.97 \\
\hline & $\mathrm{H}$ & 0.02 & 0.07 & 0.32 & 0.01 & 0.08 & 0.23 & 0.12 & 0.29 & 0.59 & 0.09 & 0.30 & 0.59 \\
\hline & I & 0.00 & 0.03 & 0.20 & 0.00 & 0.01 & 0.10 & 0.05 & 0.18 & 0.48 & 0.14 & 0.36 & 0.67 \\
\hline \multirow[t]{6}{*}{100} & D & 0.01 & 0.09 & 0.38 & 0.07 & 0.21 & 0.48 & 0.29 & 0.55 & 0.85 & 0.75 & 0.90 & 0.98 \\
\hline & $\mathrm{E}$ & 0.01 & 0.08 & 0.37 & 0.08 & 0.20 & 0.41 & 0.29 & 0.59 & 0.84 & 0.71 & 0.87 & 0.95 \\
\hline & $\mathrm{F}$ & 0.02 & 0.13 & 0.41 & 0.10 & 0.25 & 0.51 & 0.29 & 0.58 & 0.88 & 0.85 & 0.94 & 0.98 \\
\hline & $\mathrm{G}$ & 0.03 & 0.12 & 0.45 & 0.12 & 0.26 & 0.52 & 0.84 & 0.95 & 0.99 & 0.95 & 0.98 & 0.99 \\
\hline & $\mathrm{H}$ & 0.00 & 0.05 & 0.33 & 0.06 & 0.16 & 0.37 & 0.11 & 0.34 & 0.68 & 0.35 & 0.60 & 0.84 \\
\hline & I & 0.00 & 0.02 & 0.11 & 0.01 & 0.03 & 0.11 & 0.11 & 0.34 & 0.76 & 0.50 & 0.77 & 0.92 \\
\hline \multirow[t]{6}{*}{200} & D & 0.04 & 0.26 & 0.68 & 0.37 & 0.54 & 0.78 & 0.81 & 0.95 & 0.98 & 0.99 & 1.00 & 1.00 \\
\hline & $\mathrm{E}$ & 0.02 & 0.18 & 0.59 & 0.31 & 0.50 & 0.72 & 0.65 & 0.88 & 0.97 & 0.98 & 0.99 & 1.00 \\
\hline & $\mathrm{F}$ & 0.02 & 0.24 & 0.62 & 0.42 & 0.64 & 0.82 & 0.71 & 0.88 & 0.97 & 0.98 & 0.99 & 0.99 \\
\hline & $\mathrm{G}$ & 0.06 & 0.29 & 0.72 & 0.41 & 0.66 & 0.83 & 0.96 & 0.99 & 1.00 & 1.00 & 1.00 & 1.00 \\
\hline & $\mathrm{H}$ & 0.03 & 0.17 & 0.55 & 0.21 & 0.41 & 0.62 & 0.32 & 0.63 & 0.87 & 0.78 & 0.87 & 0.94 \\
\hline & I & 0.00 & 0.01 & 0.13 & 0.01 & 0.09 & 0.18 & 0.46 & 0.76 & 0.92 & 0.88 & 0.94 & 0.98 \\
\hline
\end{tabular}

Empirical true positive rates (power), for three levels of the threshold, and $N<400$.

\begin{tabular}{|c|c|c|c|c|c|c|c|c|c|c|c|c|c|}
\hline \multirow[t]{3}{*}{$N$} & \multirow[t]{3}{*}{ Cond. } & \multicolumn{6}{|c|}{ Small changes } & \multicolumn{6}{|c|}{ Large changes } \\
\hline & & \multicolumn{3}{|c|}{ AIC threshold } & \multicolumn{3}{|c|}{ BIC threshold } & \multicolumn{3}{|c|}{ AIC threshold } & \multicolumn{3}{|c|}{ BIC threshold } \\
\hline & & -15 & -10 & -5 & -15 & -10 & -5 & -15 & -10 & -5 & -15 & -10 & -5 \\
\hline \multirow[t]{6}{*}{400} & $\mathrm{D}$ & 0.26 & 0.56 & 0.88 & 0.77 & 0.88 & 0.95 & 0.98 & 1.00 & 1.00 & 1.00 & 1.00 & 1.00 \\
\hline & $\mathrm{E}$ & 0.32 & 0.67 & 0.89 & 0.80 & 0.91 & 0.97 & 0.97 & 1.00 & 1.00 & 1.00 & 1.00 & 1.00 \\
\hline & $\mathrm{F}$ & 0.33 & 0.68 & 0.90 & 0.85 & 0.92 & 0.97 & 0.98 & 1.00 & 1.00 & 1.00 & 1.00 & 1.00 \\
\hline & $\mathrm{G}$ & 0.37 & 0.72 & 0.91 & 0.88 & 0.94 & 0.97 & 1.00 & 1.00 & 1.00 & 1.00 & 1.00 & 1.00 \\
\hline & $\mathrm{H}$ & 0.09 & 0.37 & 0.78 & 0.58 & 0.79 & 0.91 & 0.67 & 0.87 & 0.98 & 0.95 & 0.97 & 0.99 \\
\hline & I & 0.01 & 0.02 & 0.22 & 0.10 & 0.22 & 0.35 & 0.91 & 0.96 & 0.99 & 0.98 & 0.99 & 1.00 \\
\hline \multirow[t]{6}{*}{600} & $\mathrm{D}$ & 0.55 & 0.83 & 0.95 & 0.92 & 0.95 & 0.97 & 1.00 & 1.00 & 1.00 & 1.00 & 1.00 & 1.00 \\
\hline & $\mathrm{E}$ & 0.58 & 0.83 & 0.98 & 0.97 & 0.99 & 1.00 & 1.00 & 1.00 & 1.00 & 1.00 & 1.00 & 1.00 \\
\hline & $\mathrm{F}$ & 0.57 & 0.80 & 0.96 & 0.92 & 0.96 & 0.97 & 1.00 & 1.00 & 1.00 & 1.00 & 1.00 & 1.00 \\
\hline & $\mathrm{G}$ & 0.59 & 0.87 & 0.96 & 0.96 & 0.98 & 0.98 & 1.00 & 1.00 & 1.00 & 1.00 & 1.00 & 1.00 \\
\hline & $\mathrm{H}$ & 0.28 & 0.61 & 0.87 & 0.78 & 0.89 & 0.93 & 0.85 & 0.96 & 1.00 & 0.99 & 0.99 & 1.00 \\
\hline & I & 0.01 & 0.08 & 0.29 & 0.19 & 0.30 & 0.44 & 0.99 & 1.00 & 1.00 & 1.00 & 1.00 & 1.00 \\
\hline \multirow[t]{6}{*}{1000} & D & 0.85 & 0.96 & 1.00 & 0.98 & 0.99 & 1.00 & 1.00 & 1.00 & 1.00 & 1.00 & 1.00 & 1.00 \\
\hline & $\mathrm{E}$ & 0.85 & 0.97 & 0.99 & 0.98 & 0.99 & 0.99 & 1.00 & 1.00 & 1.00 & 1.00 & 1.00 & 1.00 \\
\hline & $\mathrm{F}$ & 0.88 & 0.96 & 0.99 & 0.97 & 0.98 & 0.99 & 1.00 & 1.00 & 1.00 & 1.00 & 1.00 & 1.00 \\
\hline & $\mathrm{G}$ & 0.82 & 0.94 & 0.98 & 0.98 & 0.99 & 0.99 & 1.00 & 1.00 & 1.00 & 1.00 & 1.00 & 1.00 \\
\hline & $\mathrm{H}$ & 0.57 & 0.83 & 0.98 & 0.94 & 0.97 & 0.99 & 0.98 & 1.00 & 1.00 & 1.00 & 1.00 & 1.00 \\
\hline & I & 0.01 & 0.14 & 0.51 & 0.39 & 0.57 & 0.73 & 1.00 & 1.00 & 1.00 & 1.00 & 1.00 & 1.00 \\
\hline
\end{tabular}

Empirical true positive rates (power), for three levels of the threshold, and $N \geq 400$. 